\title{
SOLAR FLARE SPECTRAL DIAGNOSIS: PRESENT AND FUTURE
}

\author{
ESTER ANTONUCCI
}

Istituto di Fisica Generale dell'Università, Via P. Giuria 1, I-10125 Torino, Italy

\begin{abstract}
New perspectives in solar diagnosis have been opened in recent years with the advent of high-resolution soft X-ray spectroscopy for plasmas forming at temperatures above $10^{7} \mathrm{~K}$. The spectra obtained with the soft X-ray spectrometers flown during the last solar maximum on the major space missions dedicated to flares have allowed detailed studies of the hydrodynamic response of coronal loops to impulsive energy deposition and of the formation of the high-temperature plasma as a consequence of such dynamic effects. These studies are possible since high-resolution spectrometers give an accurate measure of both line intensities and profiles in important spectral regions, covering the emission of highly ionized heavy ions, which allow a direct determination of most of the crucial plasma parameters in the flare region. In response to the impulsive energy release in the flare region, while the intensity of soft X-ray lines increases, line profiles show large non-thermal broadenings and strong blue-asymmetries.

There have been important contributions in the understanding of the formation of the flare hightemperature plasma, as an effect of the hydrodynamic response of the solar atmosphere to impulsive chromospheric heating. On the other hand, the attempts to investigate the primary energy release and transport, on the basis of the soft X-ray spectral data, have not yet been entirely successful. Significant differences in the emitted spectra are expected at the very onset of flares for different energy deposition and transport processes, but the sensitivity of the present experiments is still insufficient to detect with good statistics the early stage of flares and, therefore, to allow a reliable discrimination. It is expected that future experiments with higher sensitivity will be of great importance for relating with less ambiguity the observed flare evolution in soft $\mathrm{X}$-rays to the primary energy deposition in the flaring coronal loops.
\end{abstract}

\section{Introduction}

The flare emission in the X-ray spectral region is classically divided into two components: a non-thermal component in hard X-rays, with photon energy above $20 \mathrm{keV}$, characterizing the impulsive phase, and a thermal component in soft X-rays, with energy below approximately $20 \mathrm{keV}$, predominant in the longer-lasting gradual phase. Soft $\mathrm{X}$-rays are emitted from an intense coronal source forming during the flare event at temperatures from 1 to $3 \times 10^{7} \mathrm{~K}$ and with an emission measure exceeding $10^{48}-10^{49} \mathrm{~cm}^{-3}$, values well above active region ones. The hot flare plasma is confined within coronal magnetic loops. Such results were primarily obtained with proportional counters (giving temperature and emission measure) and the X-ray imaging telescopes flown during the Skylab mission, which led to the identification of a highly inhomogeneous corona, with temperature and density distributions controlled by the magnetic fields.

The major advances in the study of the flare thermal plasma during solar maximum 1980 have been achieved through the study of high-temperature lines, with the advent of high-resolution spectroscopy. The emission of the flare plasma, is in fact, rich in lines of the higher ionization stages of the most abundant ions, not observed in active regions due to their lower temperatures. Three major experiments based on high-resolution

(C) 1989 Kluwer Academic Publishers. Printed in Belgium. 
crystal spectrometers were flown during solar cycle 21: the Solflex spectrometer on the P78/1 satellite, launched on February 24, 1979, covering the wavelength range 1.8-8.5 A (Doschek, 1983); the Soft X-ray Polychromator on the Solar Maximum Mission, launched on February 14, 1980, with wavelength intervals 1.4-22.4 $\AA$ and 1.7-3.3 $\AA$ (Acton et al., 1980); and the SOX spectrometers on the Hinotori satellite, launched on February 26, 1981, with wavelength range 1.73-1.95 $\AA$ (Tanaka et al., 1982a). The most important spectral regions selected for these experiments include the $\mathrm{H}$-like and He-like emission of heavy ions, such as $\mathrm{Ca}$ and $\mathrm{Fe}$. The Fexxv region has been observed by all three experiments. This important line group was first observed by Neupert et al. (1967) and spectrally resolved by Grineva et al. (1973). Such observations made possible the identification of the individual lines in the group. High-resolution spectroscopy of flare emission has offered the possibility to determine directly most of the thermal plasma parameters and to measure the dynamics in the flare region.

\section{Diagnosis of the Thermal Flare Plasma}

The study of the intensity of the hot flare lines in the spectral regions of emission of $\mathrm{H}$-like and He-like ions of heavy elements gives information on the plasma by allowing determination of physical parameters such as electron temperature, differential emission measure, ionization states and, in some cases, electron density and element abundances. In addition to the line-intensity diagnosis, high-resolution spectrometers have provided, for the first time, through the measure of line profiles and thereby of Doppler temperatures and Doppler shifts, a complete picture of mass motions in the flare region. The observations have shown that the flare thermal plasma is highly dynamic at least in the early phase of the event.

\subsection{Diagnostic methods for the He-like ions}

The spectral regions of the He-like ions are rich in satellites to the He-like resonance lines, falling at relatively longer wavelengths, formed by dielectronic recombination of the He-like ions with electrons and by inner-shell excitation of Li-like ions, which become observationally important for high nuclear charge ions. Satellites give the possibility of a direct measure of both the electron temperature and the departure from ionization equilibrium of the flaring plasma. The electron temperature is measured as a function of the relative satellite to resonance line intensity in a manner which is independent of ionization balance, since the lines are coming from the same ionization stage. A similar method based on the ratio of the satellite to resonance line intensity can also be used for H-like ion spectra (Dubau et al., 1981; Parmar et al., 1981). The ionization equilibrium is tested by measuring, for instance, the relative intensity of the He-like to the Li-like spectrum, which is a function of the relative populations of adjacent ionization stages. The importance of dielectronic recombination satellites for directly measuring plasma parameters has been pointed out and the theory has been developed by Gabriel (1972), Bhalla, Gabriel, and Presnyakov (1975) and later works of Gabriel and co-workers. The ratio of the forbidden to intercombination lines of 

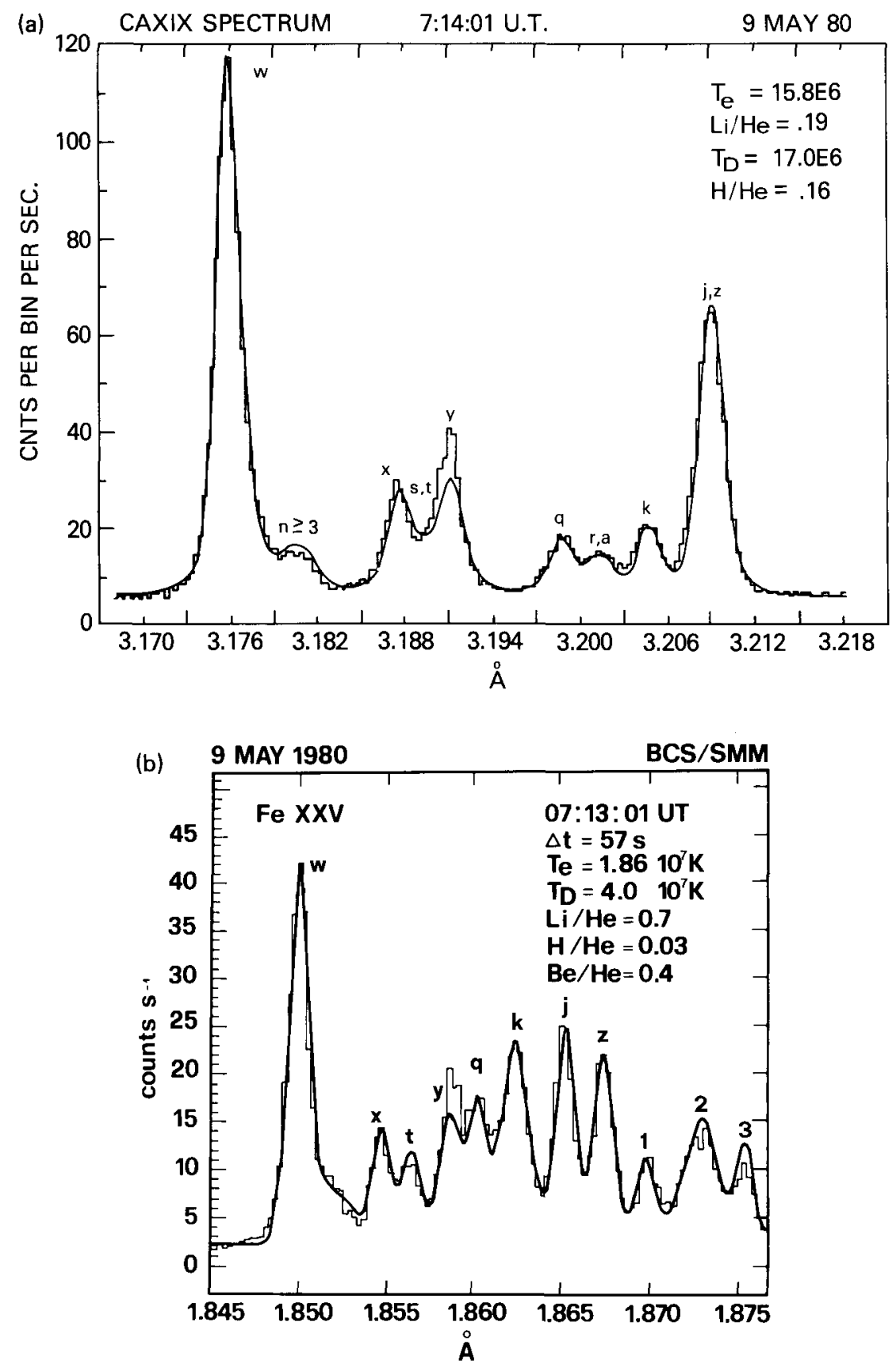

Fig. 1. CaxIX (a) and Fexxv (b) spectra observed with the Soft X-Ray Polychromator on the Solar Maximum Mission. The continuous curves represent spectra best fitting the observations. 
He-like ions are instead sensitive to electron density (Gabriel and Jordan, 1969). The ions sensitive to flare coronal densities, such as OvII, NeIX, MgXI, are, however, formed at lower temperatures (with emissivity peaking in the interval from 2 to $\left.6 \times 10^{6} \mathrm{~K}\right)$ than those characterizing the hotter soft X-ray source $\left(10^{7} \mathrm{~K}\right)$ during flares.

\subsection{Temperature MEASUREMENTS}

The He-like and $\mathrm{H}$-like ion emissions more extensively observed during flares in the course of the 1980 solar maximum are relative to CaxIX, Caxx, FeXXv, and FexXvi. Figures 1(a) and 1(b) present an example of the He-like $\mathrm{Ca}$ and $\mathrm{Fe}$ ion emission, respectively. Plasma parameters are usually derived by fitting a theoretical spectrum to the observed one (Antonucci et al., 1982; Akita, 1985; Tanaka, 1986). This method is used to take into proper account the heavy line blending occurring in this zone. The values of electron temperature derived from the emission in the spectral regions of different ions may differ when the temperature distribution in the flare plasma departs significantly from isothermal conditions. The temperature derived from a line ratio even in the same spectral region is, in fact, a mean value of the temperature distribution averaged with the line contribution functions, which in the case of hightemperature X-ray lines spread over a large temperature interval.

Temperature measurements with the SOX/Hinotori observations in FeXXV and Fe XXVI have evidenced the existence of a 'hot', or 'superhot', dense thermal component, with temperature $T_{e}$ above $3 \times 10^{7} \mathrm{~K}$ and density $n_{e}$ above $10^{11} \mathrm{~cm}^{-3}$, in half of the large flares observed (Tanaka et al., 1982a; Tsuneta et al., 1984; Tanaka, 1986). The discovery of such 'hot thermal flares' confirms previous results obtained with hard X-ray continuum by Lin et al. (1981). 'Hot thermal flares' are characterized by a close association of the temporal evolutions in the FexxvI intensity and hard X-ray continuum below $40 \mathrm{keV}$, and a departure of the temperature derived from the $\mathrm{H}$-like FexxvI, $T_{e}(\mathrm{H})$, from that obtained from the He-like Fexxv, $T_{e}(\mathrm{He})$, around the peak of the gradual phase. $T_{e}(\mathrm{H})$ and $T_{e}(\mathrm{He})$ are instead the same during the rising and decaying phase, Figure 2 (Tanaka, 1986). The gradual character of the hard X-ray emission observed below $40 \mathrm{keV}$ is not maintained above such a value. At higher energies impulsive spikes are detected, as shown in Figure 3 (Tsuneta, 1987). The 'hot' thermal plasma seems to be confined in a compact source high in the loop (Takakura et al., 1983; Tanaka, 1986).

Such observational results have been interpreted as an indication that, in the highdensity 'hot' thermal source, the low energy cutoff of non-thermal electrons shifts to higher energies. That is, in the dense 'hot-thermal flares', acceleration of low-energy electrons becomes less efficient than in flares characterized by a lower density. This has led to the suggestion that the density in the energy release site plays an important role in partitioning the released energy into particle acceleration and local heating (Tanaka, 1987; Tsuneta, 1987; Watanabe, 1987). 


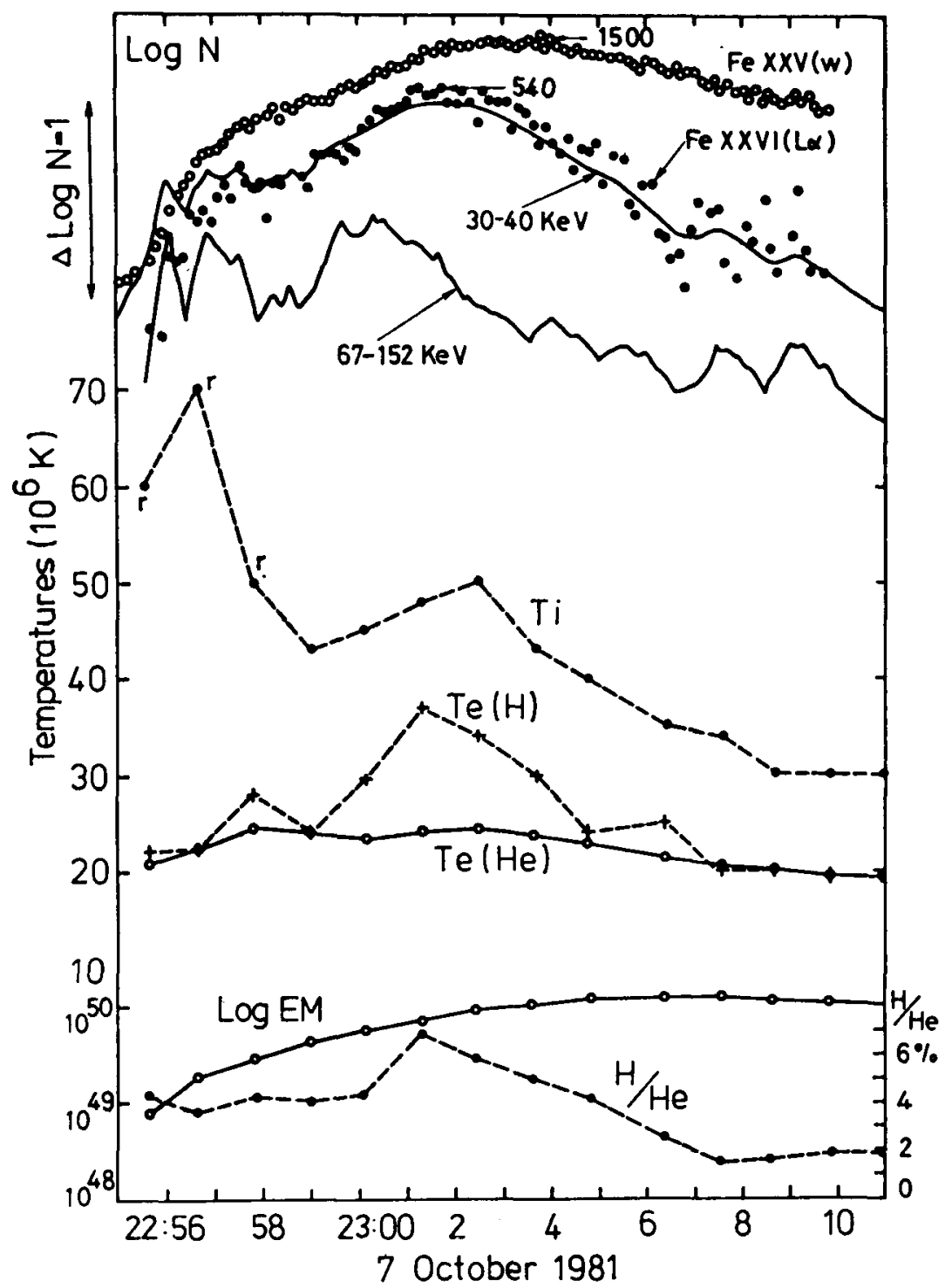

Fig. 2. Evolution of the physical parameters derived from the Fexxv and Fexxvi Hinotori observations for a 'hot thermal flare' (Tanaka, 1986).

\subsection{DENSITY MEASUREMENTS}

Flare densities have been measured from the He-like spectra of O VII, Ne IX, and MgXI. McKenzie et al. (1980) and Doschek et al. (1981) derived from the O viI emission values of $n_{e}=3 \times 10^{12} \mathrm{~cm}^{-3}$ for the April 8, 1980 flare. From NeIX spectra, Wolfson et al. (1983) derived values ranging from $3 \times 10^{11} \mathrm{~cm}^{-3}$ to $1.5 \times 10^{12} \mathrm{~cm}^{-3}$ in the November 5, 1980 flare. In Mg XI spectra, Linford and Wolfson (1989) and Linford, Lemen, and Strong (1989) measured values of about $3 \times 10^{12} \mathrm{~cm}^{-3}$ for a sample of 
1981 APRIL 2

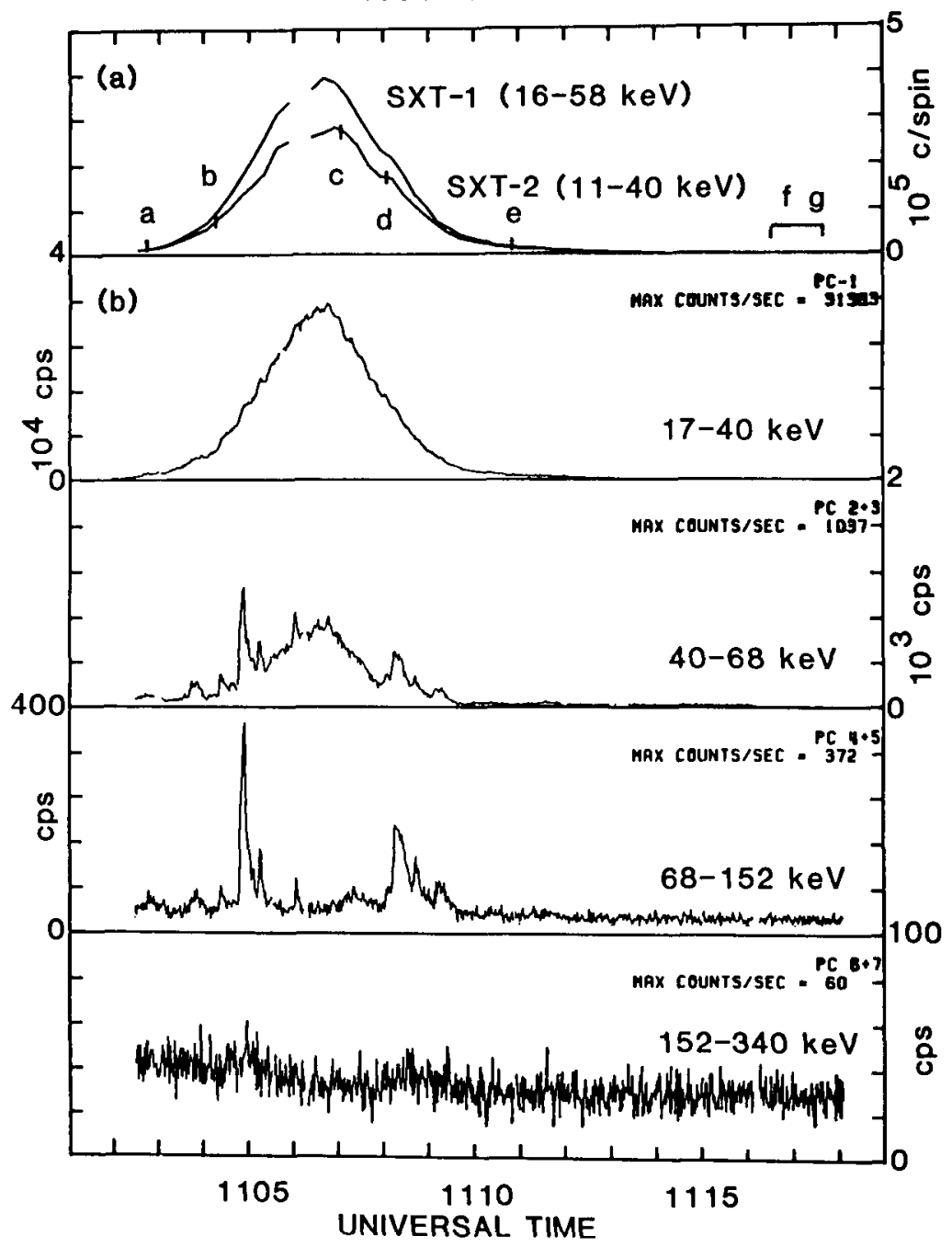

Fig. 3. Evolution of the hard X-ray emission in a 'hot thermal flare' (Tsuneta, 1987).

flares including the May 21, 1985 event. Such direct measurements give densities which are in general higher than those inferred from the emission measure combined with information on the geometry of the flaring region. This discrepancy may be due to the fact that the ions sensitive to flare densities are emitted from a lower temperature soft X-ray source; while emission measure and geometry are usually given for a plasma at higher temperature. Such a discrepancy can, however, also suggest that the flare plasma is confined in very thin loops (e.g., Linford and Wolfson, 1989) and, therefore, the flare region is characterized by filamentary unresolved elements. The filling factor derived for the flares studied in density-sensitive emission lines ranges from $3 \times 10^{-4}$ (Linford and Wolfson, 1989) to $10^{-2}$ (Wolfson et al., 1983). These values are consistent with filling 
factors derived for soft X-ray loops in non-flaring conditions on the basis of scaling law considerations (Martens, van den Oord, Hoyng, 1985) and for active regions in the phase immediately preceding the flare onset by studying the flow velocities in the reconnection sites (Antonucci, Rosner, and Tsinganos, 1986).

\section{Dynamics in the Flare Region}

The soft X-ray emission, which is evidence for the formation of the thermal plasma at temperatures above $10^{7} \mathrm{~K}$, traditionally defines the flare gradual or thermal phase; while the hard X-ray emission is associated with the impulsive phase. The highresolution observations of line profiles have, however, shown that soft X-rays also have impulsive phase signatures. The soft $\mathrm{X}$-ray line profiles are in fact extremely sensitive in monitoring, although indirectly, the onset and evolution of the flare impulsive energy release. The two distinctive features of soft $\mathrm{X}$-ray lines during the impulsive phase are large non-thermal broadenings, enhanced above the active region values, and a significant blue-shifted emission (Antonucci et al., 1982). Figure 4 shows the temporal evolution of the soft and hard X-ray emission and compares typical impulsive and gradual phase CaXIX spectra. Gradual phase profiles are generally thermal, or quasi-thermal; that is, line broadenings are consistent with the average electron temperature derived from line ratios in the same spectral region. These observations of soft $\mathrm{X}$-ray line profiles clearly show that the formation of the flare thermal plasma, at $1-3 \times 10^{7} \mathrm{~K}$, is associated with the presence of high-velocity mass motions.

While non-thermal broadenings are interpreted as evidence for isotropic flows, or random motions, in the flare region, blue-shifts are evidence for the hydrodynamic response of the chromosphere to the impulsive energy release. In flare conditions, the chromosphere heats up to high temperatures either by thermal flux from a hot overlying coronal source or by a flux of non-thermal particles accelerated in the energy release site. The energy deposited at chromospheric level causes an expansion of the plasma, as a result of heating, which gives rise to high velocity upflows into the lower density corona: that is, to chromospheric evaporation. Their onset is roughly simultaneous with the onset of the hard X-ray emission indicating impulsive energy release. In most of the events with a detectable soft $X$-ray emission before the impulsive phase, non-thermal broadenings in soft X-ray lines are observed to precede blue shifts (Figure 5).

\subsection{NON-THERMAL LINE BROADENINGS}

Most of the interpretations relate the origin of the enhanced non-thermal line broadenings observed during the impulsive phase to the presence of convective flows, associated with chromospheric evaporation. Non-thermal broadenings have been explained as a result of a superposition of emissions from convective flows observed either in an arcade of flaring loops with different projection angles (Doschek et al., 1986), or in an asymmetrically heated loop, as in the model of Cheng, Karpen, and Doschek (1984). They have also been interpreted as a result of a velocity distribution in the evaporating plasma which is not entirely accounted for by analysing soft X-ray spectra simply in terms of 


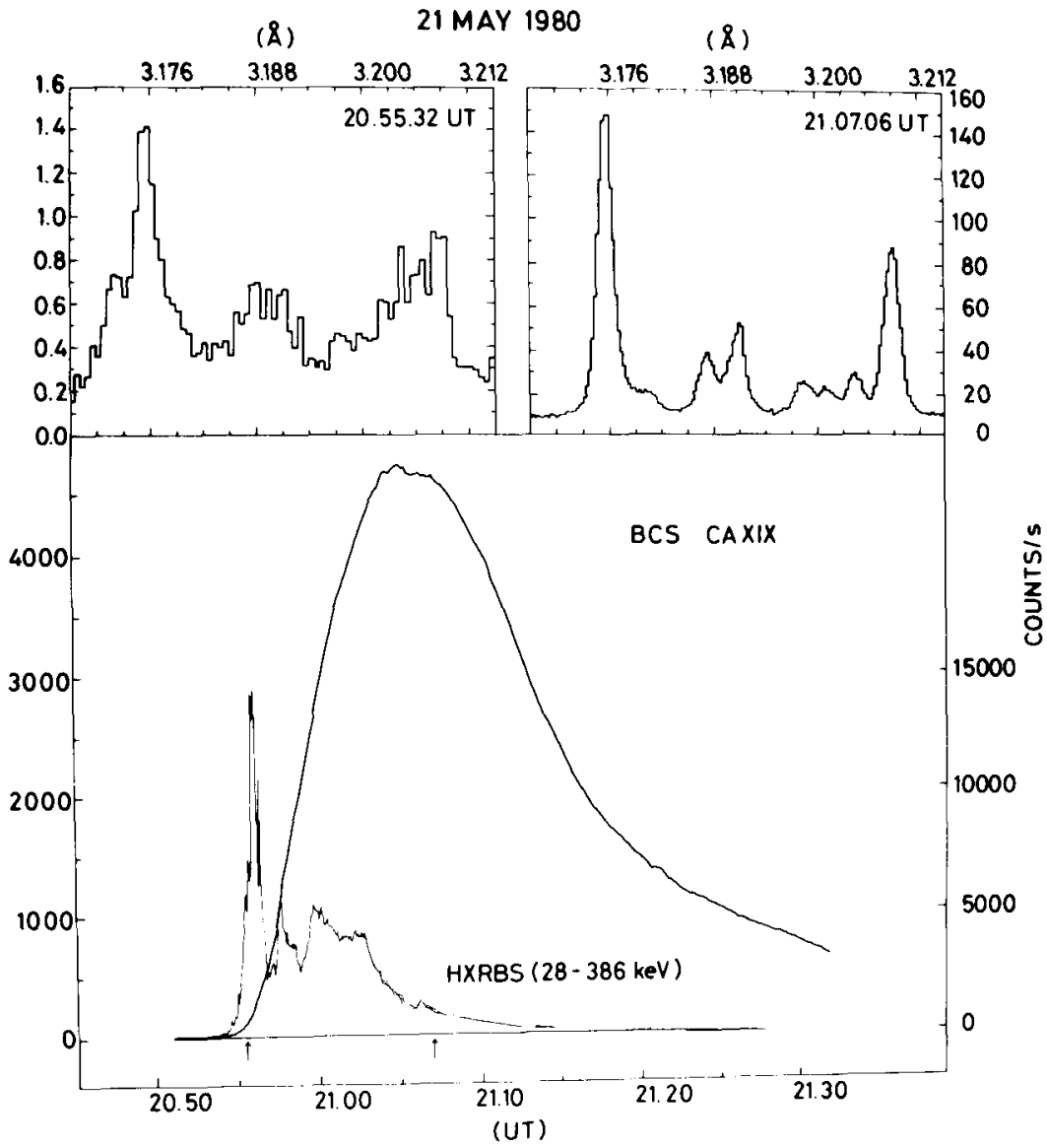

Fig. 4. Ca XIX spectra during the impulsive and gradual phase of the May 21, 1980 flare observed with the Soft X-ray Polychromator on the Solar Maximum Mission satellite, at the top. At the bottom, light curves of the soft and hard X-ray emission during the same event.

two components (Emslie and Alexander, 1987). Other interpretations, which relate enhanced broadenings to the evaporation process in a more indirect way, have also been proposed. Bornmann (1987) proposes an interpretation in terms of fluid turbulence, in which the kinetic energy of upflows transforms into thermal energy. De Jager (1985) interprets line broadenings as evidence for a temperature difference at the sites where evaporation occurs.

Although all these interpretations are plausible, they cannot predict the observed enhancement in line widths preceding the onset of blue-shifts. The non-thermal excess in soft X-ray line profiles increases significantly above the active region values already a few minutes before the onsets of the impulsive hard X-ray burst, that is of the impulsive energy release, and of evaporation (which are approximately coincident). In addition, no strong further enhancement in line broadening is observed when convective flows are first observed. As an example, if the degree of non-thermal broadening is expressed 


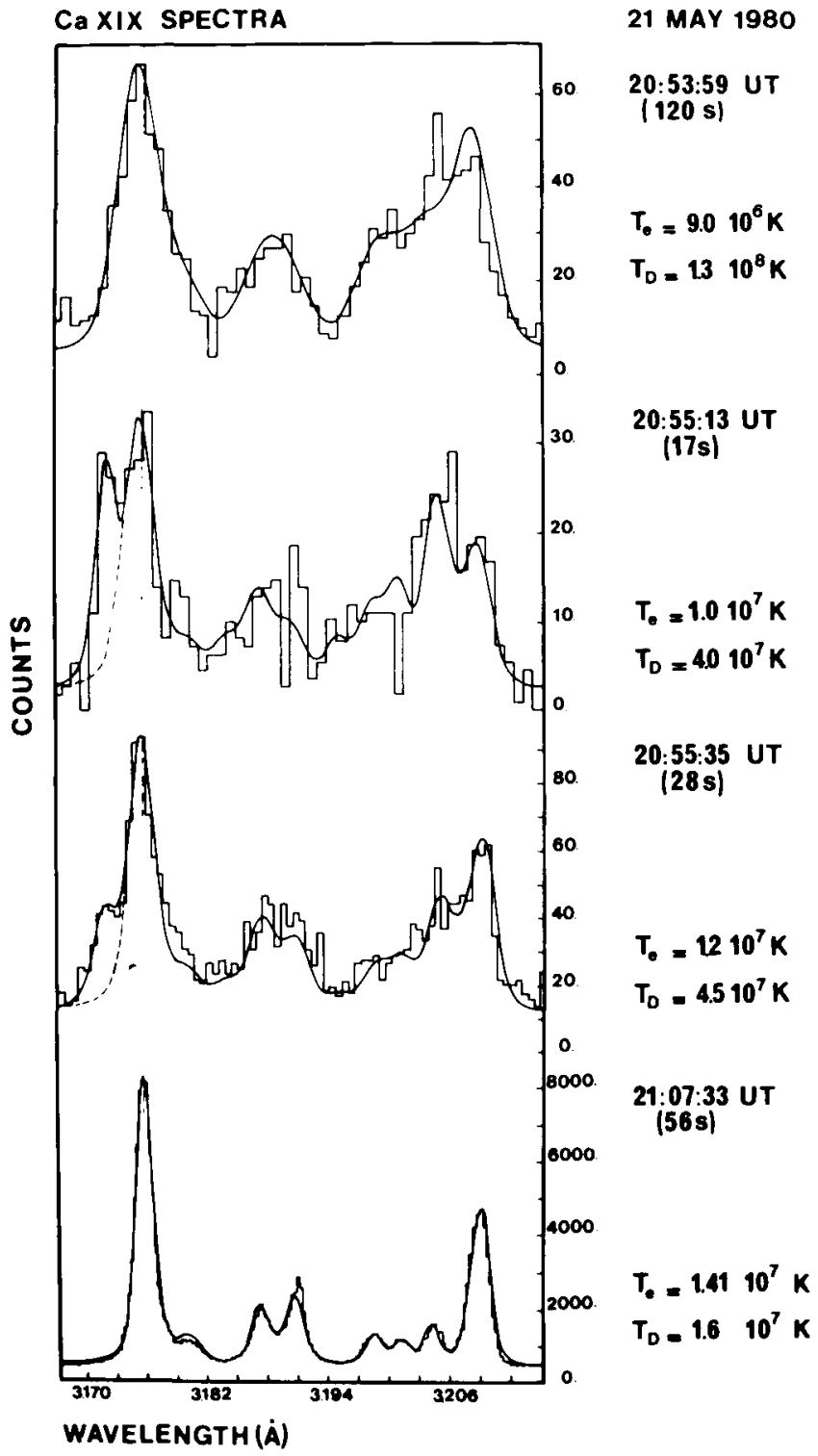

Fig. 5. Temporal sequence for the onset of enhanced non-thermal line broadenings and blue-shifts in soft X-rays during the May 21, 1980 flare. In the CaxIX spectrum detected at 20:53:59 UT, the difference in Doppler temperature $T_{\mathbf{D}}$ and electron temperature $T_{e}$ is consistent with a turbulent velocity parameter $v_{t}=220 \mathrm{~km} \mathrm{~s}^{-1}$. An intense blue-shifted emission is first detected at 20:55:13 UT. The spectrum becomes thermal at the end of the impulsive phase, approximately in coincidence with the peak of the gradual phase

(Antonucci et al., 1985).

in terms of a turbulent velocity parameter, $v_{t}=\left[2 k\left(T_{\mathrm{D}}-T_{e}\right) / m\right]^{1 / 2}$ (where $T_{\mathrm{D}}$ is the Doppler temperature and $T_{e}$ is the electron temperature obtained from line ratio), no evident variation in $v_{t}$ is associated with the time $\mathrm{T}$, indicating the onset of blue shifts in CaXIX during the large April 24, 1984 flare (Figure 6). Therefore, although both 


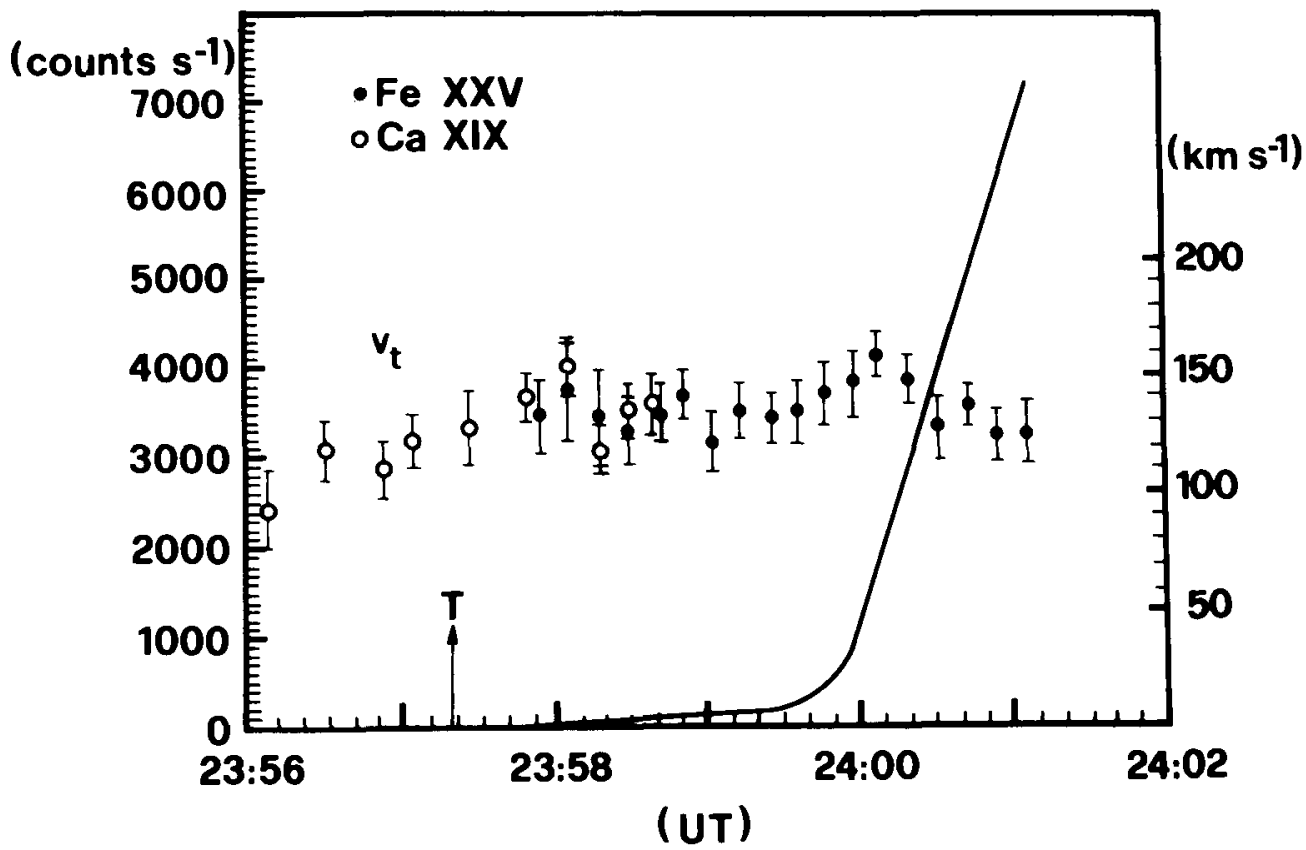

Fig. 6. Evolution of turbulent velocity, $v_{t}$, during the early phase of the April 241984 flare (class X13, importance 3B), observed with the Soft X-ray Polychromator on SMM. Light symbols represent the values derived from the CaXIX line profiles, while dark symbols refer to FexXv data. The continuous line represents the Caxix light curve. The time $\mathrm{T}$ represents the onset of upflows, evidence for chromospheric evaporation (Antonucci and Dodero, 1986).

non-thermal broadenings and upflows are present throughout the impulsive phase, and vanish or decrease to a minimum level at the end of it, the onset and degree of broadening are not significantly correlated with convective upflows.

Furthermore, if non-thermal widths were a result of a superposition of convective flows, they should be larger in disk flares, since vertical flows are predominant in evaporation. However, no such center-to-limb variation has been found with the present accuracy in the measure of turbulent velocity $\left(v_{t}=100 \pm 40 \mathrm{~km} \mathrm{~s}^{-1}\right.$ for disk flares and $v_{t}=120 \pm 30 \mathrm{~km} \mathrm{~s}^{-1}$ for limb flares).

The consistency of turbulent velocities derived from CaXIX and Fe XXV data, shown in Figure 6, confirms that the difference in Doppler and electron temperatures does not represent a real ion-electron temperature difference, which cannot be maintained for time intervals of minutes in a plasma at coronal flare densities.

The implication is, therefore, that enhanced non-thermal broadenings are evidence for random or 'turbulent' mass motions. Furthermore, such motions seem to be related to the onset of energy release, although not yet in an impulsive form. The initial enhancement in broadenings is, in fact, temporally correlated with the appearance of lowintensity hard X-ray emission which precedes the main burst. This indicates an initial phase of particle acceleration, associated with an initial temperature increase in the soft $\mathrm{X}$-ray source. Turbulent velocities are, however, already very high and do not change 
appreciably when energy release becomes impulsive; that is, the degree of enhancement in turbulent velocity does not appear to be directly related to the degree of energy released.

A plausible explanation can be found by interpreting random plasma flows as a dynamic effect related to the magnetic reconnection process. In particular, it is possible that reconnection takes place in localized regions scattered throughout the flaring loop. This picture is consistent with coronal loops whose magnetic field lines become twisted by photospheric motions, leading then to local reconnection, with consequent magnetic energy dissipation throughout the loop (Antonucci, Rosner, and Tsinganos, 1986; Parker, 1987). Plasma is ejected at the Alfvén speed from a localized site, where magnetic reconnection occurs (Sweet, 1969; Parker, 1983). An isotropic flow results then from the superposition of individual flows and causes symmetric broadenings in soft X-ray lines (Antonucci, Rosner, and Tsinganos, 1986, 1987). In this hypothesis, an enhancement in line broadening occurs simultaneously with the onset of magnetic energy dissipation in the flare region and persists as long as the process continues. The same process suggested to initiate a flare can also be invoked to heat the quiet corona and active regions. Concerning the latter point, interesting observations of enhanced non-thermal excess in line profiles have been obtained by Saba et al. (1989) along the more intense magnetic fields, in an active region.

\subsection{IMPULSIVE PHASE BLUE-SHIFTS AND CHROMOSPHERIC EVAPORATION}

The properties of the soft X-ray line blue-shifts derived from studies of the impulsive phase of a large sample of flares strongly suggest that blue-shifts are evidence for chromospheric evaporation (Antonucci et al., 1982; Tanaka et al., 1982b; Antonucci and Dennis, 1983; Antonucci, Gabriel, and Dennis, 1984; Tanaka, 1986). Flows with velocities within $100-500 \mathrm{~km} \mathrm{~s}^{-1}$ are consistent with motions which are predominantly ascensional, since blue-shifts are not observed in flares occurring beyond approximately $60 \mathrm{deg}$ in longitude (Antonucci, Gabriel, and Dennis, 1984; Tanaka, 1986). Such upflows are associated both spatially and temporally with an impulsive heating of the chromosphere. Their onset and duration is correlated with the impulsive hard X-ray emission, which indicates that energy is released in the flare region (Figure 7). At the time upflows are first observed, the soft $\mathrm{X}$-ray emission between 3.5 and $8 \mathrm{keV}$ is found to be co-spatial with the hard X-ray emission in the $16-30 \mathrm{keV}$ range (within the resolution of the Hard X-Ray Imaging Spectrometer on SMM), and confined to the footpoints of the flaring loops (Figure 8).

The duration of upflows is associated with the rise in the emission from the hot coronal source forming during flares, since blue-shifts persist so long as the emission measure of the stationary component of the soft X-ray source is increasing. This is to be expected if the evaporating plasma is confined in magnetic loops. In addition, blue-shifts decrease while the emission from the thermal source increases. This can be understood in terms of a progressive decrease in the pressure difference between chromosphere and corona while the loop density increases, which causes a progressive decrease in evaporation velocities (Figure 9). 


\section{MAY 1980}

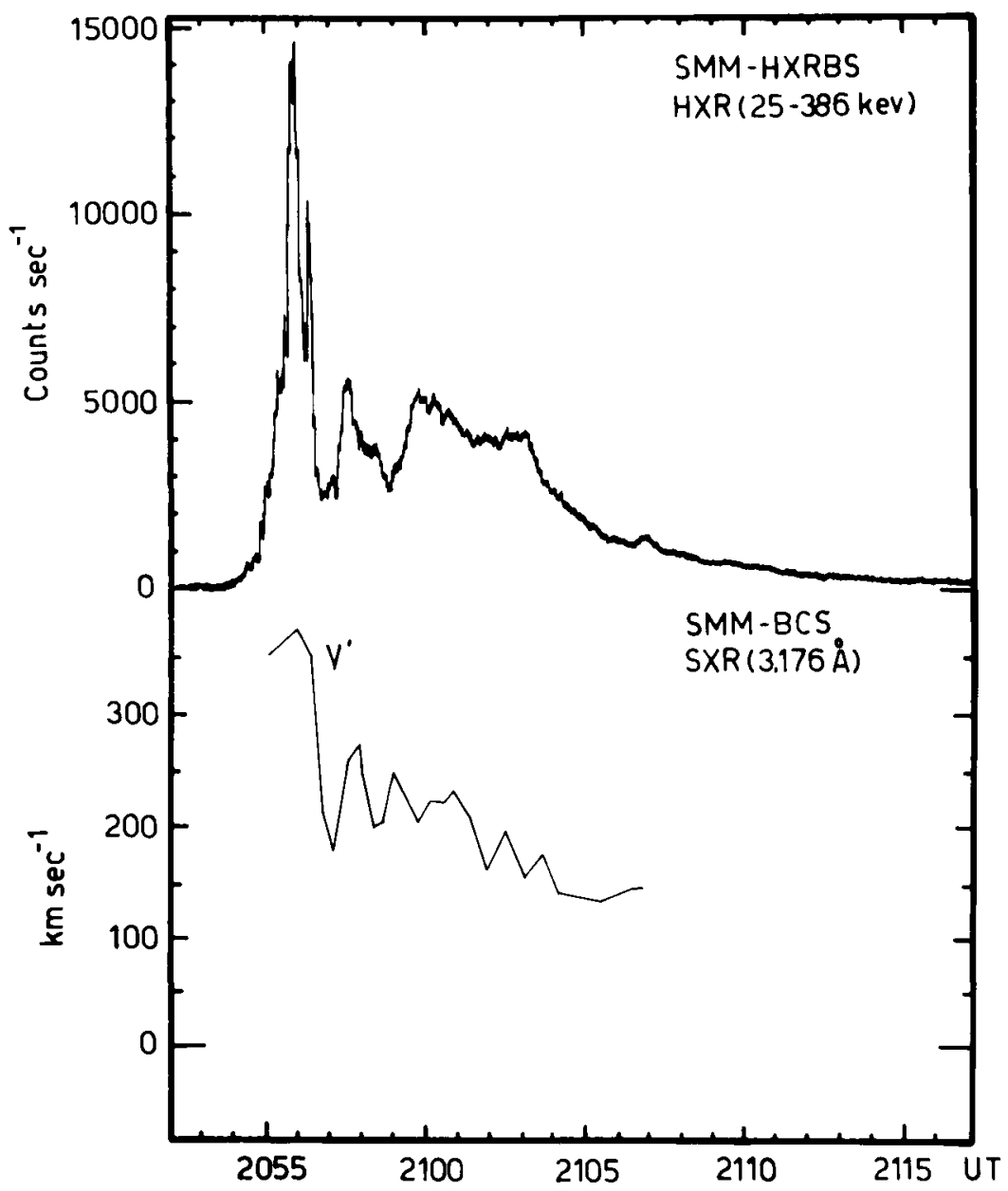

Fig. 7. Temporal evolution of the velocities, $v^{\prime}$, derived for the upflows due to chromospheric evaporation and the impulsive emission in hard X-rays, signaling energy release, in the May 21, 1980 flare.

If the upflowing material due to chromospheric evaporation plays a dominant role in the formation of the thermal coronal plasma in flares, we expect a mass and energy balance between the evaporating material and the soft X-ray coronal source. Moreover, since density and temperature of the evaporating plasma cannot be directly measured due to the blending of the temperature diagnostic lines emitted from the evaporating and coronal plasmas, we can only assert that mass and energy balance can be ensured for plausible values of such quantities: that is, densities of the order of $1-2 \times 10^{11} \mathrm{~cm}^{-3}$, and temperatures of $1-2 \times 10^{7} \mathrm{~K}$ (Antonucci et al., 1982; Antonucci, Gabriel, and Dennis, 1984). Densities derived from mass balance represent lower limits, mainly because of the unresolved geometry of the flare region.

By following the same approach and requiring mass and energy balance during the 
$3.5-8 \mathrm{keV}$

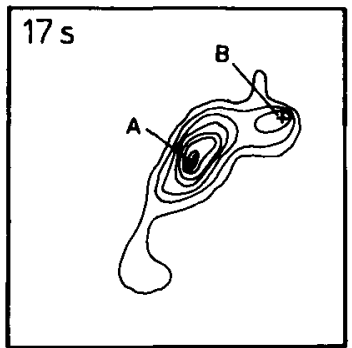

\section{MAY 1980}
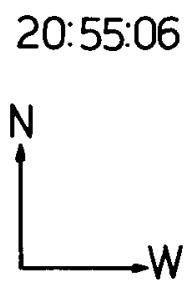

\section{$16-30 \mathrm{keV}$}

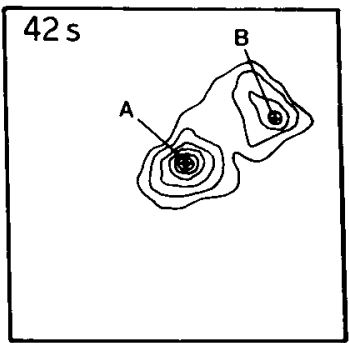

Fig. 8. Example of footpoint brightening in soft and hard X-rays at the onset of chromospheric evaporation for the May 21, 1980 flare, as observed by the SMM experiments (Antonucci et al., 1985).

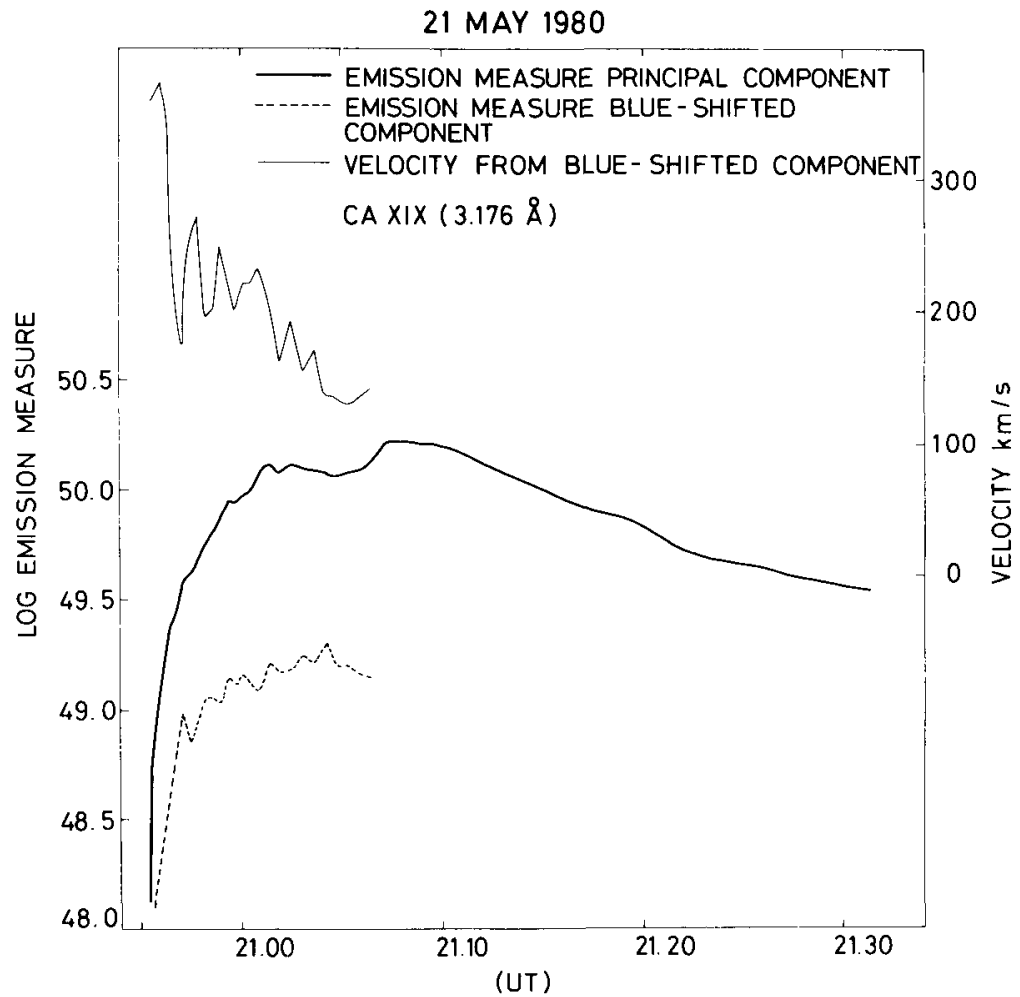

Fig. 9. Temporal evolution of the chromospheric evaporation velocity and the emission measure of the thermal soft X-ray source forming in the corona during the May 21, 1980 flare. The emission measure of the upflowing plasma is also reported as a function of time.

flare impulsive phase between upflowing and coronal plasmas, Tanaka and Zirin (1985) found the observations of the large June 6, 1982 flare consistent with the evaporation interpretation. The same procedure, however, applied to the large November 7, 1980 flare led Karpen, Doschek, and Seely (1986) to conclude that the amount of the 
upflowing plasma is in excess, or in other words, the upflowing plasma does not have sufficient pressure, according to the inferred density value, to expand into the coronal portion of the loop. The geometry of this flare, however, contrary to the other events studied by Antonucci, Gabriel, and Dennis (1984) and Tanaka and Zirin (1985) is totally unresolved. In general, because of the impossibility of resolving the individual sites where evaporation occurs and the difficulties in determining the effective pre-flare density in the flaring loops, we can only derive a lower limit for the density of upflows.

\subsection{COMPARISON OF OBSERVATIONAL PROPERTIES AND SIMULATION RESUlTS}

We can investigate whether the general properties of plasma upflows are consistent with the predictions of the numerous hydrodynamical models developed in recent years to understand the hydrodynamical response of a coronal loop undergoing transient impulsive heating. For example, independently of the model assumed for energy deposition and heat input, at the onset of chromospheric evaporation the soft X-ray emission is expected to be predominant at the footpoints of flaring loops, as observed (e.g., Figure 8). This is consistent with the simulations shown in Figure 10. CaxIX brightenings at the footpoints of a loop undergoing transient heating are predicted both for energy deposited by an electron beam injected at the top of the loop (which heats predominantly the chromosphere via Coulomb losses), or in the hypothesis of a thermal energy source located either at the apex or at the base of the loop (with consequent chromospheric heating by thermal conduction). Only for thermal energy deposition high in the corona, are the footpoint brightenings expected to be delayed with respect to the onset of flare heating, with a time lag consistent with the transit time of the thermal conduction front (Antonucci et al., 1987).

The average parameters, simulated both for the evaporating plasma and for the thermal source which is forming by magnetic confinement at coronal level, shown in Figures 11 and 12, reproduce the general temporal behavior of the parameters derived from the observed soft X-ray emission (Figure 9). The relative velocity of the upflowing material with respect to the coronal soft X-ray source, $v^{\prime}$, decreases with time although the energy input flux remains constant for the entire interval considered $(180 \mathrm{~s})$. Both the intensity of the coronal source $I_{w}$ and of the upflowing material, $I_{w}^{\prime}$ (Figures 11 and 12) are steadily increasing during such an interval (although $I_{w}^{\prime}$ decreases relative to the emission of the static soft X-ray source), as observed in Figure 9. Considering the relatively low-energy input flux, $\phi=6-8 \times 10^{9} \mathrm{erg} \mathrm{cm}^{-2} \mathrm{~s}^{-1}$ used in these calculations, the line intensities simulated at flare onset are too weak to be observable with an instrument with sensitivity such as that of the Bent Crystal Spectrometer (Antonucci et al., 1987).

The fact that model predictions in general indicate a dominant blue-shifted emission at flare onset, which is not systematically observed with the present instrumentation, has raised some questions about whether impulsive phase blue-shifts are indeed evidence for chromospheric evaporation (Doschek et al., 1986; Emslie and Alexander, 1987). In fact, if the energy input flux is increased above $10^{10}-10^{11} \mathrm{erg} \mathrm{cm}^{-2} \mathrm{~s}^{-1}$, the simulated blue-shifted emission is expected to dominate the coronal plasma emission, at flare 

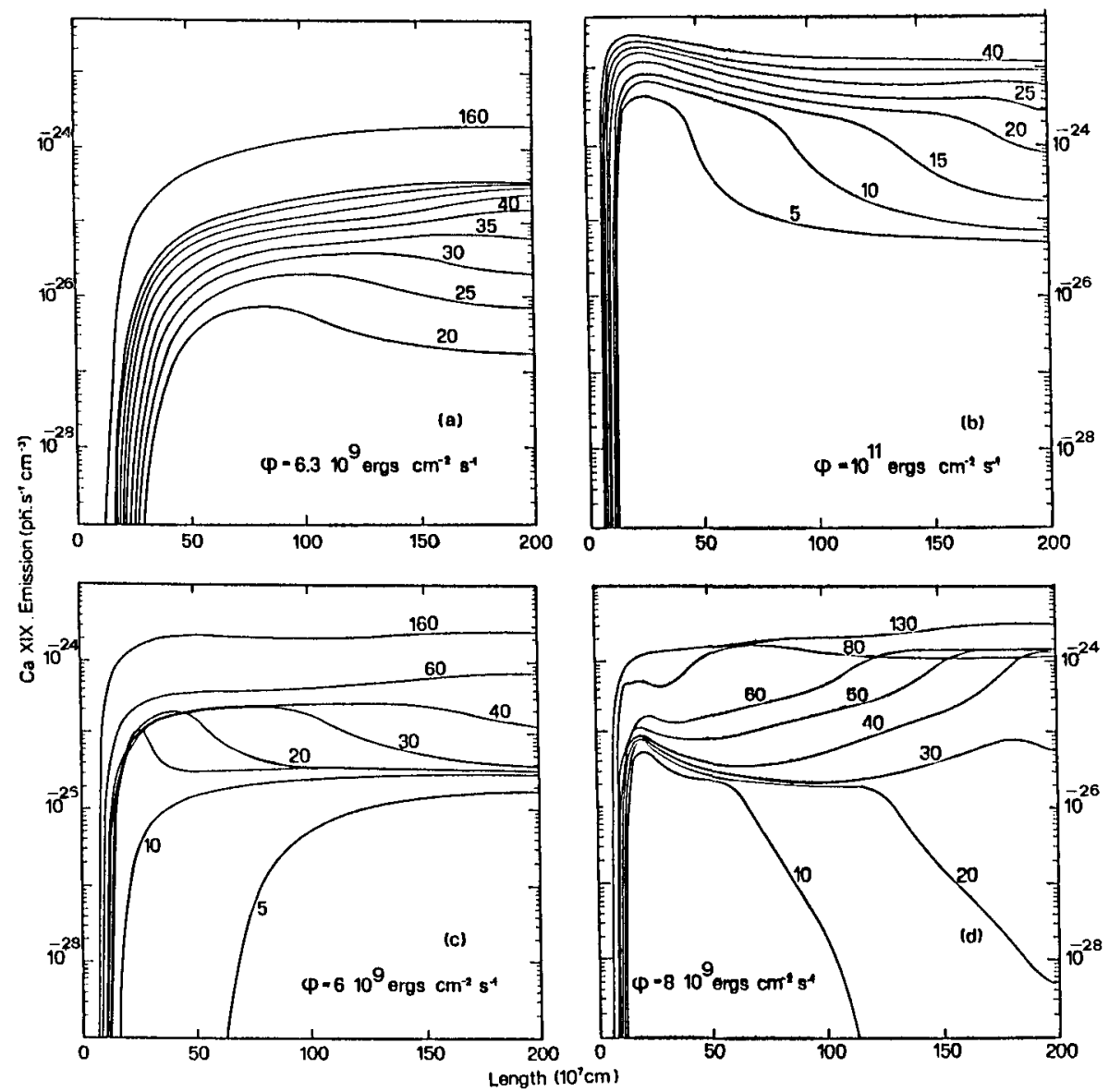

Fig. 10. Simulated curves of CaXIX emission versus height in a flaring loop of semi-length $l / 2=2 \times 10^{9} \mathrm{~cm}$, initial density $n_{e}=7 \times 10^{9} \mathrm{~cm}^{-3}$, initial temperature $T_{e}=3.2 \times 10^{6} \mathrm{~K}$, for different energy deposition models: (a) injection of an electron beam parallel to the field lines, confining the onedimensional fluid, with input energy flux of $6.3 \times 10^{9} \mathrm{erg} \mathrm{cm}^{-2} \mathrm{~s}^{-1}$ (with a power-law energy spectrum with lower cutoff $E_{c}=10 \mathrm{keV}$ and spectral index $\delta=8$ ), (b) injection of an electron beam with the same characteristics as in the previous case but with higher energy flux $\phi=10^{11} \mathrm{erg} \mathrm{cm}^{-2} \mathrm{~s}^{-1}$, (c) thermal energy deposition at the top of the loop with energy input flux $\phi=6 \times 10^{9} \mathrm{erg} \mathrm{cm}^{-2} \mathrm{~s}^{-1}$, (d) thermal energy deposition at the base of the loop with $\phi=8 \times 10^{9} \mathrm{erg} \mathrm{cm}^{-2} \mathrm{~s}^{-1}$. The simulations are performed with the unidimensional, one-fluid Palermo-Harvard hydrodynamical model (Antonucci et al., 1987).

onset, even more strongly than for the moderate energy fluxes used in the models of Figures 11 and 12. The dominant blue-shifted emission is presumably not commonly observed, since at flare onset the instrumental time resolution is degraded in order to improve the statistics of the spectra. A recent re-analysis by Tanaka (1987) of the large June 6, 1982 flare observed with the Hinotori spectrometers has shown that all the emission in FeXXV is initially blue-shifted and the shift is consistent with velocities of the order of $300 \mathrm{~km} \mathrm{~s}^{-1}$ (Figure 13, frame a). A static coronal soft X-ray source, 


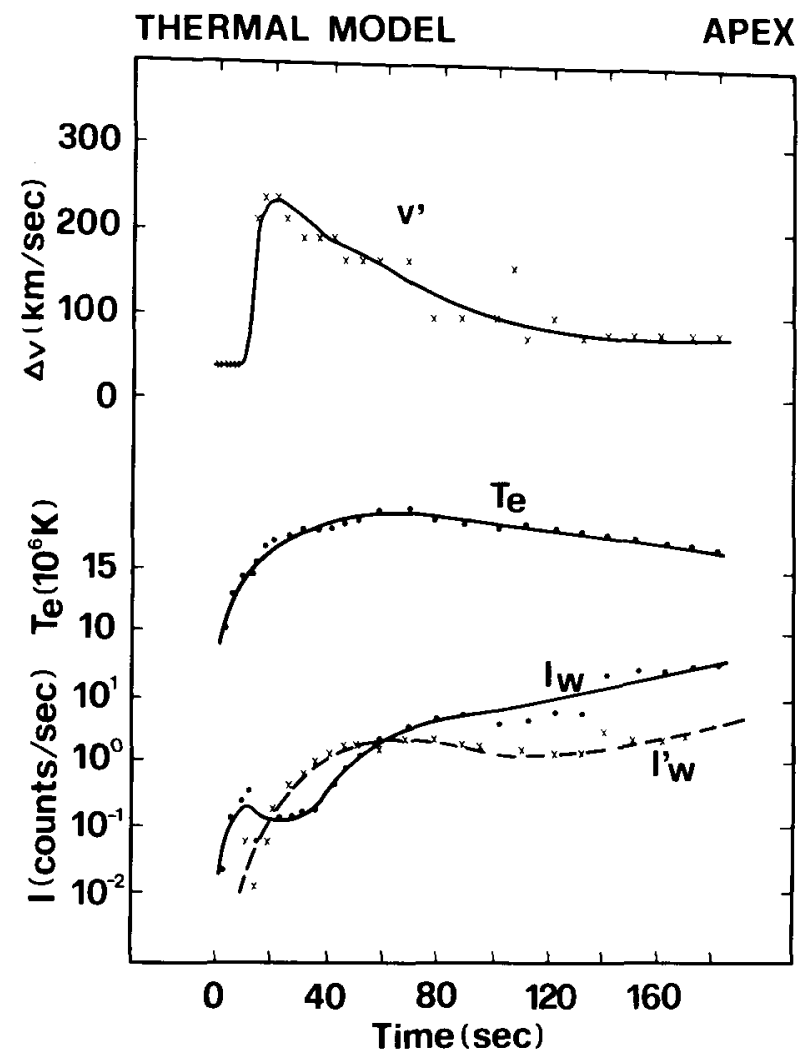

Fig. 11. Evolution of the simulated average relative velocities of the evaporating plasma, $v^{\prime}$, the average electron temperature in the forming coronal thermal plasma, $T_{e}$, the CaXIX intensities, $I_{w}$ and $I_{w}^{\prime}$ of the coronal and upflowing plasma emission, respectively. These parameters are derived for a flaring loop with energy deposited as in case (c) of Figure 10, with thermal heating at the top of the loop at a constant rate for an interval of $180 \mathrm{~s}$. The quantities $v^{\prime}, T_{e}, I_{w}$, and $I_{w}^{\prime}$, are derived from simulated Ca XIX spectra, computed on the basis of the physical conditions predicted along the loop, in response of the flare heating. $I_{w}$ and $I_{w}^{\prime}$ are expressed in counts $\mathrm{s}^{-1}$, expected in the CaxIX channel of the Bent Crystal Spectrometer of SMM

(Antonucci et al., 1987).

forming presumably by confinement of the evaporating plasma, appears at the rest wavelength of Fexxv after approximately $40 \mathrm{~s}$, and continues to grow afterwards (frames $\mathrm{c}$ and d of Figure 13). Only later on (frames e and f), a typical impulsive phase spectrum with emission of the static source predominant with respect to blue emission is observed.

Figure 14 shows an example of simulation of the evolution of CaxIX spectra for a model with a thermal energy source localized at the footpoints of a flaring loop. Spectral profiles evolve showing initially the development of a dominant blue-shifted component. After about $35 \mathrm{~s}$ however, the emission from the coronal source forming by confinement of evaporating plasma in closed field lines becomes predominant. This evolution is similar to that observed for the June 6, 1982 flare by Tanaka (1987). 


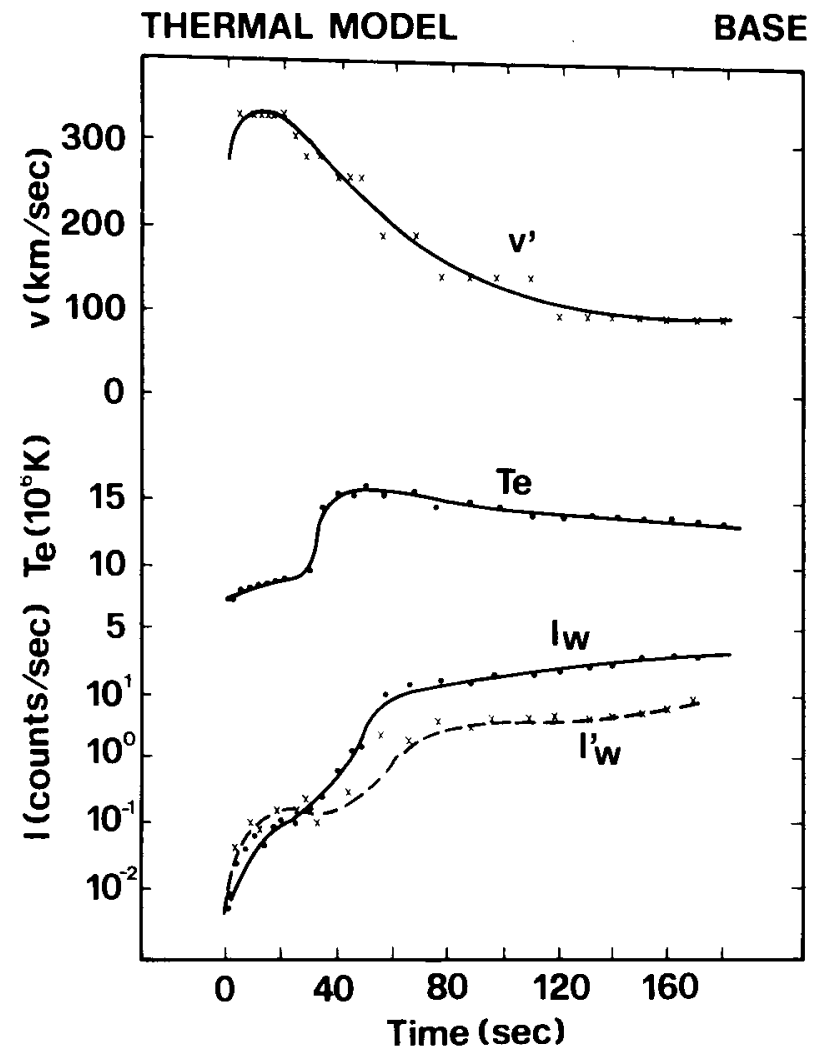

Fig. 12. Flare plasma parameters, as in Figure 11, but for thermal heating at the base of the loop (case (d) of Figure 10) (Antonucci et al., 1987).

Since the simulations of Figure 14 have been derived in the case of relatively moderate energy flux input, they give initially very low counting rates, which are, for instance, below the sensitivity of an instrument such as the Bent Crystal Spectrometer (SMM), unless photons are accumulated for intervals of about $40 \mathrm{~s}$ (Figure 15). In this case, the typical profile of an impulsive phase spectrum is obtained. This explains how instrumental sensitivity can be responsible for observing a dominant blue emission at the very onset of chromospheric evaporation.

\section{Additional Evidence for Chromospheric Evaporation}

As a sufficiently strong beam of non-thermal electrons loses its energy in the chromosphere, the sudden temperature increase creates explosive chromospheric evaporation. In explosive conditions, the local pressure excess produces not only an upward 

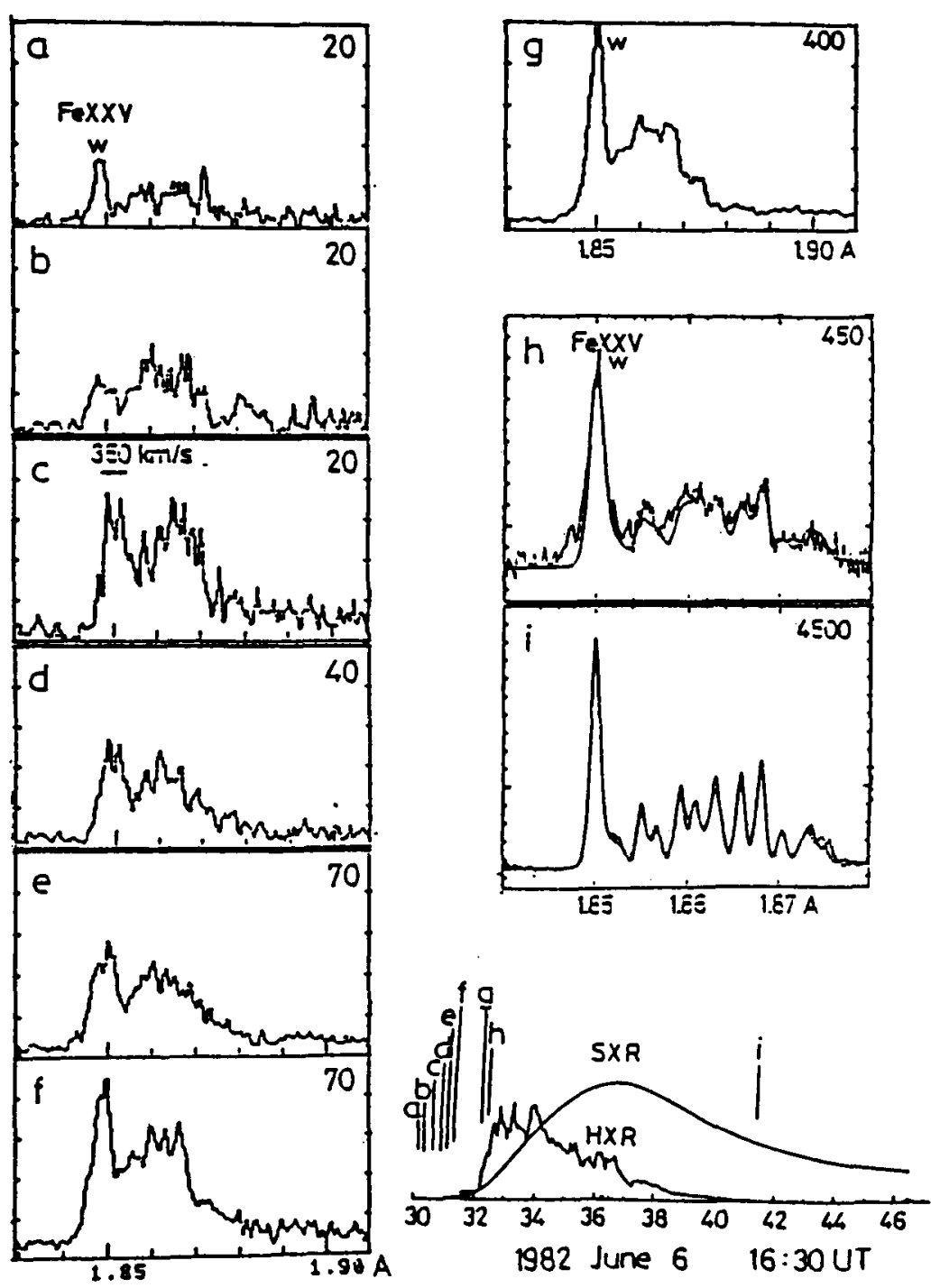

Fig. 13. Time sequence of Fe XxV spectra during the June 6, 1982 flare. The emission at $1.85 \AA$ shows at first the development of a blue-shifted dominant resonance line (frame $a, b, c, d$ ), and the formation of the coronal source emitting an unshifted component at $1.85 \AA$ (since frame b), which becomes predominant after about $100 \mathrm{~s}$ (frame e, f). The wavelengths are calibrated in reference to the $\mathrm{K} \alpha$ line at $1.94 \AA$ (Tanaka, 1987;

Watanabe, 1987, review).

expansion into the pre-flare corona, observed as blue-shifted soft X-ray emission, but it also produces a compression of the underlying unevaporated material driving a pressure wave down into the chromosphere, which can be observed as red-shifted $\mathrm{H} \alpha$ emission. At the same time, the signature expected for non-thermal electrons entering the chromosphere is the presence of broad Stark wings in $\mathrm{H} \alpha$ (Canfield, Gunkler, and 


\section{SIMULATED SPECTRAL EMISSION}
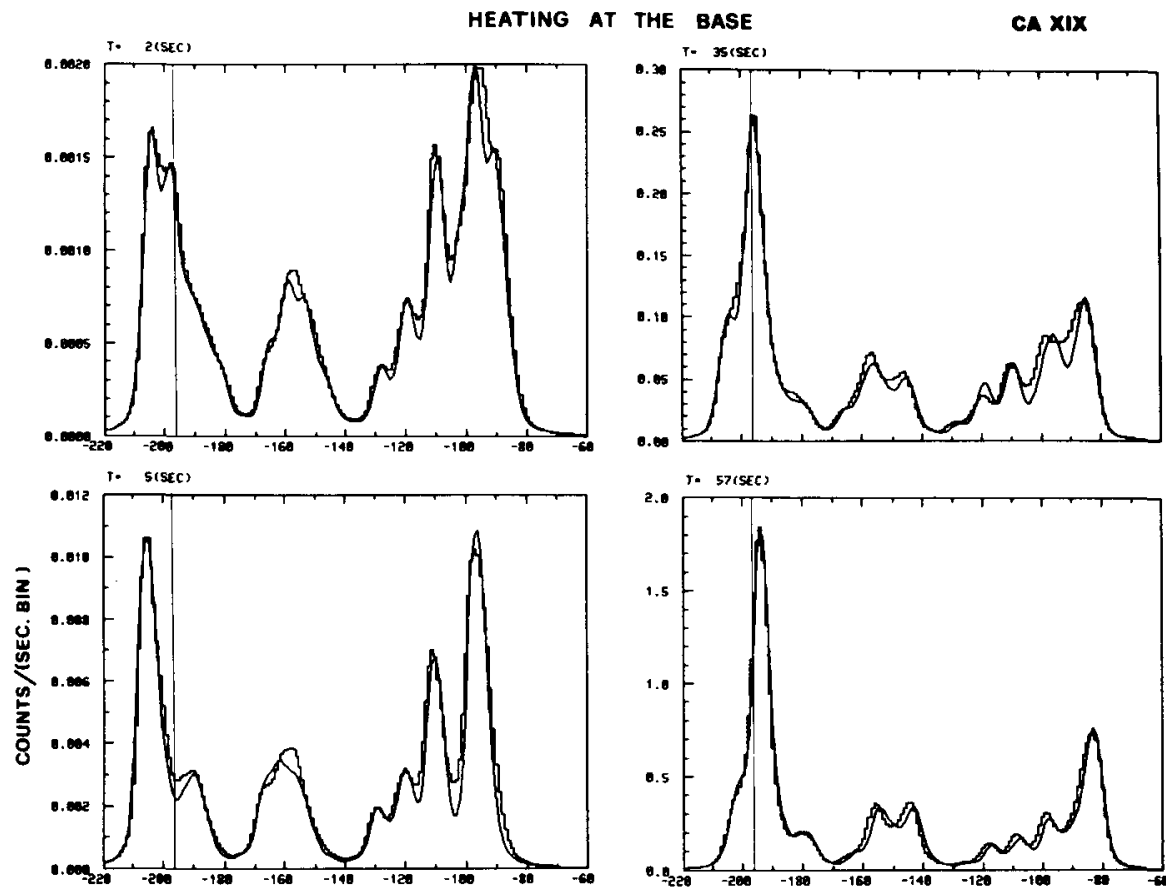

T. $39(\sec )$
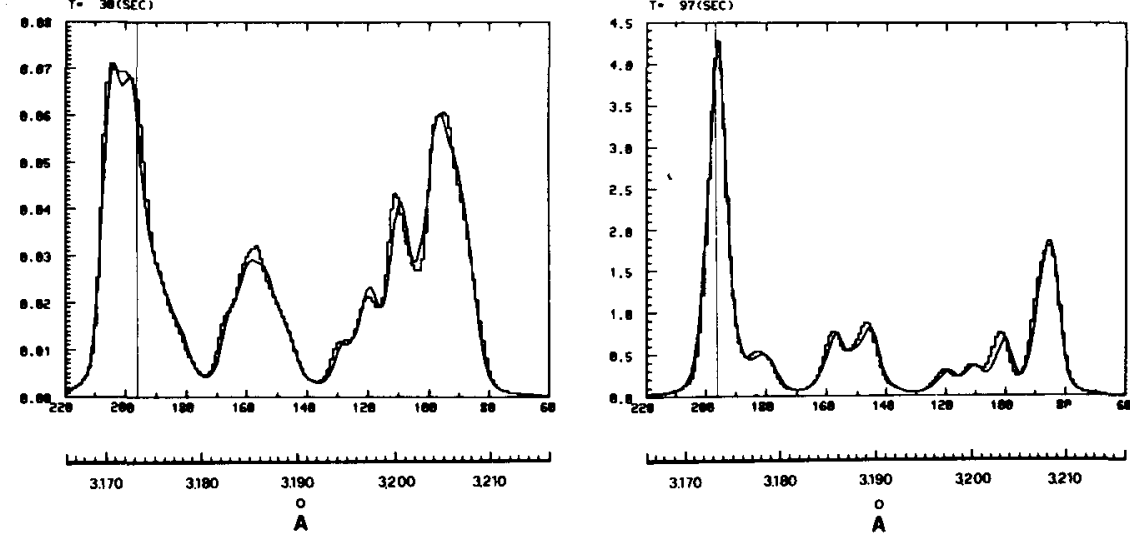

Fig. 14. Evolution of the simulated CaXIX spectra for a thermal model of energy deposition localized at the base of the loop, as in Figure 10(d). The vertical line in each plot represents the unshifted CaXIX resonance line at $3.176 \AA$. The intensity is in units of counts $\mathrm{s}^{-1}$ bin $^{-1}$ of the CaXIX channel of the Bent Crystal Spectrometer on SMM. The continuous lines represent theoretical two-component synthetic spectra fitted to the simulated spectra (stepped curves) (Antonucci et al., 1987).

Ricchiazzi, 1985). Evidence for broad Stark wings due to electron beams and red-shifts in $\mathrm{H} \alpha$ (Ichimoto and Kurokawa, 1984; Canfield, 1986) have indeed been found in coincidence with the flare impulsive hard X-ray emission. The observed downward velocities, within $40-100 \mathrm{~km} \mathrm{~s}^{-1}$, and lifetime of the chromospheric condensation are consistent with predictions from the simulations by Fisher, Canfield, and McClymont 


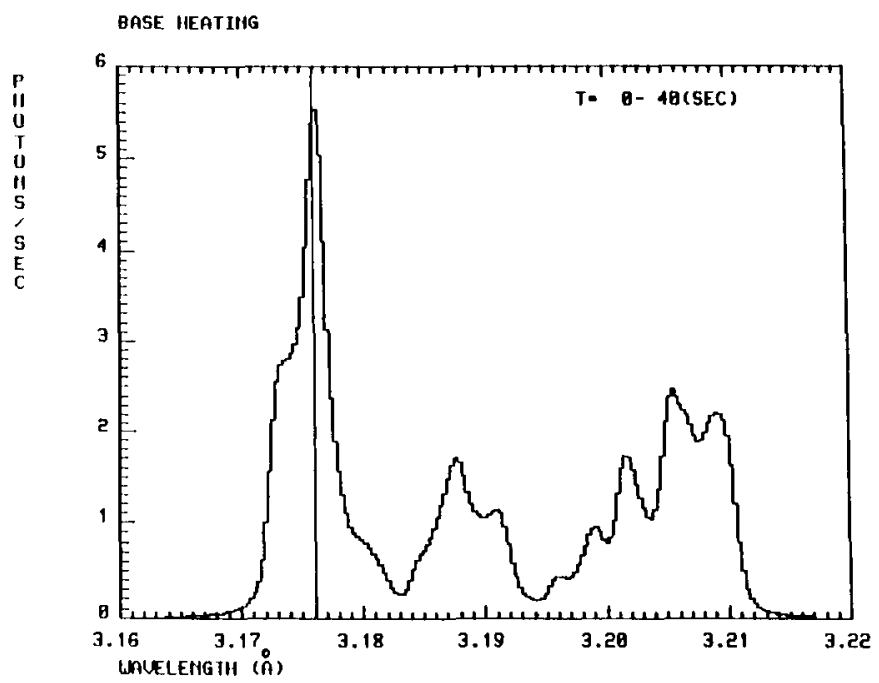

Fig. 15. CaxIX simulated spectrum integrated over the initial $40 \mathrm{~s}$ for a thermal model with energy deposition localized at the base of the loop, as in Figure 10(d).

(1985). In addition, evidence for the appearance of soft X-ray emitting plasma associated with a simultaneous disappearance of $\mathrm{H} \alpha$ chromospheric material was found by Acton et al. (1982) and Gunkler et al. (1984).

While it has been possible to investigate, at least indirectly, the mass and energy balance between upflows and the forming coronal source during the impulsive phase, uniquely on the basis of soft X-ray data (Antonucci, Gabriel, and Dennis, 1984), simultaneous observations in soft X-rays and in $\mathrm{H} \alpha$, obtained in a few flares, have recently led Canfield et al. (1989) to ascertain, within the uncertainties of the present measurements, momentum balance between the evaporating soft X-ray plasma and the downflowing $\mathrm{H} \alpha$ chromospheric plasma. The column density at which chromospheric motions, upflows and downflows, are expected to commence is found to be consistent with the theoretical stopping depth of a beam of non-thermal electrons in a thick target (Zarro et al., 1989).

Lower temperature emission lines also give additional evidence for chromospheric evaporation during the impulsive phase of flares. During the impulsive phase of three flares, an increase in the volume of the O VII source (forming at $2 \times 10^{6} \mathrm{~K}$ ) has been observed in temporal correlation with the blue-shifts in higher temperature lines. This has been interpreted as an expansion of the source resulting from chromospheric evaporation (Bruner et al., 1988).

In a single well-resolved loop observed in Fexv (emitted at temperature below $10^{7} \mathrm{~K}$ ) during the Skylab mission, Hiei and Widing (1979) reported a progressive brightening from the footpoint to the top of the loop. This phenomenon as well can be interpreted in terms of chromospheric evaporation. 


\section{Alternative Interpretative Models for Impulsive Soft X-Ray Blue-Shifts}

There have been several attempts to explain soft X-ray blue-shifts during the impulsive phase of flares not simply in terms of the hydrodynamic response of coronal loops to a transient impulsive energy deposition, but by considering them as evidence for magnetohydrodynamic forces acting at the onset of flares. Karpen, Doschek, and Seely (1986) propose a magnetohydrodynamic model, where a sudden heating of twisted flux-tubes results in gas expulsion. Plasma flows away from the solar surface toward regions of weaker magnetic field at a velocity equivalent to the proton thermal velocity of a few $10^{2} \mathrm{~km} \mathrm{~s}^{-1}$.

In a model of reconnecting loops (Doschek et al., 1986), upflows can be created within a lower density loop colliding with an emerging dense flux-tube. In this case, upflows are simply driven by the pressure difference between the two flux-tubes.

Uchida and Shibata (1988) propose a flare model based on untwisting loops. Upflows are expected in the unwinding process occurring when a twisted flux-tube emerges from the photosphere in a lower pressure region, where the plasma pressure can no longer dominate the magnetic force. In the process, plasma heated to soft X-ray temperatures is ejected predominantly upward.

Upflows in flares may be also due to coronal ions accelerated in a direction perpendicular to the magnetic field and mirrored upward in convergent flux-tubes. This effect is found to occur in a bi-dimensional model which considers the filamentation in an electron beam current (Winglee, Pritchett, and Dulk, 1988).

At the present such alternative explanations of soft X-ray blue-shifts cannot predict more than the dominant direction of the plasma motions as well as a general temporal association of the onsets of upflows and impulsive phase. They cannot, however, predict the spatial association of soft X-ray emission with footpoints at flare onset; the temporal relation of upflows to the increase in emission of the high-temperature plasma; and the decrease in upflow velocity when the density of the soft X-ray source is increasing. In addition, none of these models explains either the existence of oppositely directed flows: the upward hot evaporating material emitting soft X-rays and the downward cold chromospheric condensation observed in $\mathrm{H} \alpha$ nor the momentum balance of upflowing and downflowing plasmas, as discussed by Canfield (1986) and Canfield et al. (1989).

\section{X-Ray Blue-Shifts as Signature of Chromospheric Heating}

We have discussed the X-ray blue-shifts as evidence for chromospheric evaporation and their role in the formation of the high-temperature plasma (above $10^{7} \mathrm{~K}$ ), observed during flares. The question is whether the characteristics of the impulsive blue-shifted emission in soft X-ray lines can also be studied to investigate the primary energy release and the role of different mechanisms in heating the chromosphere to high temperature.

Several numerical models have been developed to simulate the hydrodynamic response of the plasma confined in a coronal magnetic loop to an impulsive and intense energy release. These models show that the properties of evaporation depend not only 
on the parameters characterizing energy release and on the process which heats the chromosphere, but also on the initial conditions and geometry of the loop. Explosive evaporation occurs for chromospheric heating by intense particle beams (e.g., Somov, Syrovatskii, and Spektor, 1981; Nagai and Emslie, 1984; MacNeice et al., 1984; Fisher, Canfield, and McClymont, 1985) or by intense thermal fluxes (e.g., Smith and Harmony, 1982; Somov, Sermulina, and Spektor, 1982; MacNeice, 1986), with values above a few $10^{10} \mathrm{erg} \mathrm{cm}^{-2} \mathrm{~s}^{-1}$. This is due to the fact that heating takes place on a time-scale shorter than the hydrodynamic expansion time-scale of the plasma. Therefore, evaporation velocity can be considered as a signature of the flux of energy released.

Evaporation velocities derived from the CaxIX line profiles, which have been extensively studied, are typically within $100-500 \mathrm{~km} \mathrm{~s}^{-1}$ with average peak value around $300 \mathrm{~km} \mathrm{~s}^{-1}$ (Antonucci, Gabriel, and Dennis, 1984). Velocities derived from the Fe XXV line profiles show a tendency to give higher values (Feldman et al., 1980; Antonucci et al., 1982; Karpen, Doschek, and Seely, 1986; Tanaka, 1987). A recent study of $\mathrm{FexXv}$ line profiles detected in the high-sensitivity, high-spectral resolution channel of the Bent Crystal Spectrometer on the Solar Maximum Mission, shows that the velocity distribution in the evaporating plasma extends to much higher values. The plasma component evaporating at higher velocity is characterized by a relatively weaker emission and a higher average temperature with respect to the bulk of the evaporating plasma (Antonucci, Dodero, and Martin, 1989).

For example, the profile of the CaXIX resonance line observed during the impulsive phase of the extremely intense April 24, 1984 flare can be reproduced by a superposition of two synthetic spectra, whose relative wavelength shift is consistent with a line-of-sight velocity of $210 \mathrm{~km} \mathrm{~s}^{-1}$ (Figure 16). The simultaneous profile of the Fe XXV resonance line (Figure 17(a)), observed in the high-sensitivity channel of the Bent Crystal Spectrometer (SMM), shows a weak but significant blue excess with respect to the fit consisting of two spectral components, which are Doppler-shifted in agreement with the velocity observed in CaXIX. Such an excess in blue-emission is evidence for plasma evaporating on the average at a line-of-sight velocity of $480 \mathrm{~km} \mathrm{~s}^{-1}$ (Figure 17(b)).

The analysis of a set of 11 large flares, of class $\mathrm{M}$ and $\mathrm{X}$, has shown that very high velocities are often observed in the early impulsive phase. The average velocity $v_{2}$ for the excess blue emission in FeXXV is found to be within $500-800 \mathrm{~km} \mathrm{~s}^{-1}$; while, the velocity $v_{1}$ relative to the upflows observed also in CaXIX for the same set of flares is within $250-400 \mathrm{~km} \mathrm{~s}^{-1}$. Maximum values in the velocity distribution, $v_{2, \max }$, defined on the basis of the shift corresponding to $\lambda_{2, \max }^{\prime}$ where the intensity decreased by $e^{-1}$ (Figure 17(b)), are within 700-1000 $\mathrm{km} \mathrm{s}^{-1}$.

The ratio of the intensities of the emission in CaXIX and Fe XXV from the evaporating plasma moving at average velocity $v_{1}$ (first blue-shifted spectral component in Figures 16 and $17(\mathrm{~b})$ ) gives the possibility to estimate directly the average temperature for such a plasma. For the plasma evaporating at velocity $v_{2}$, observed only in Fexxv, it is, instead, possible to estimate just a temperature lower limit which results from the ratio of the second blue-shifted spectral component in Fexxv and the background intensity (at the correspondent wavelength shift) in Caxix. The temperature derived for the 


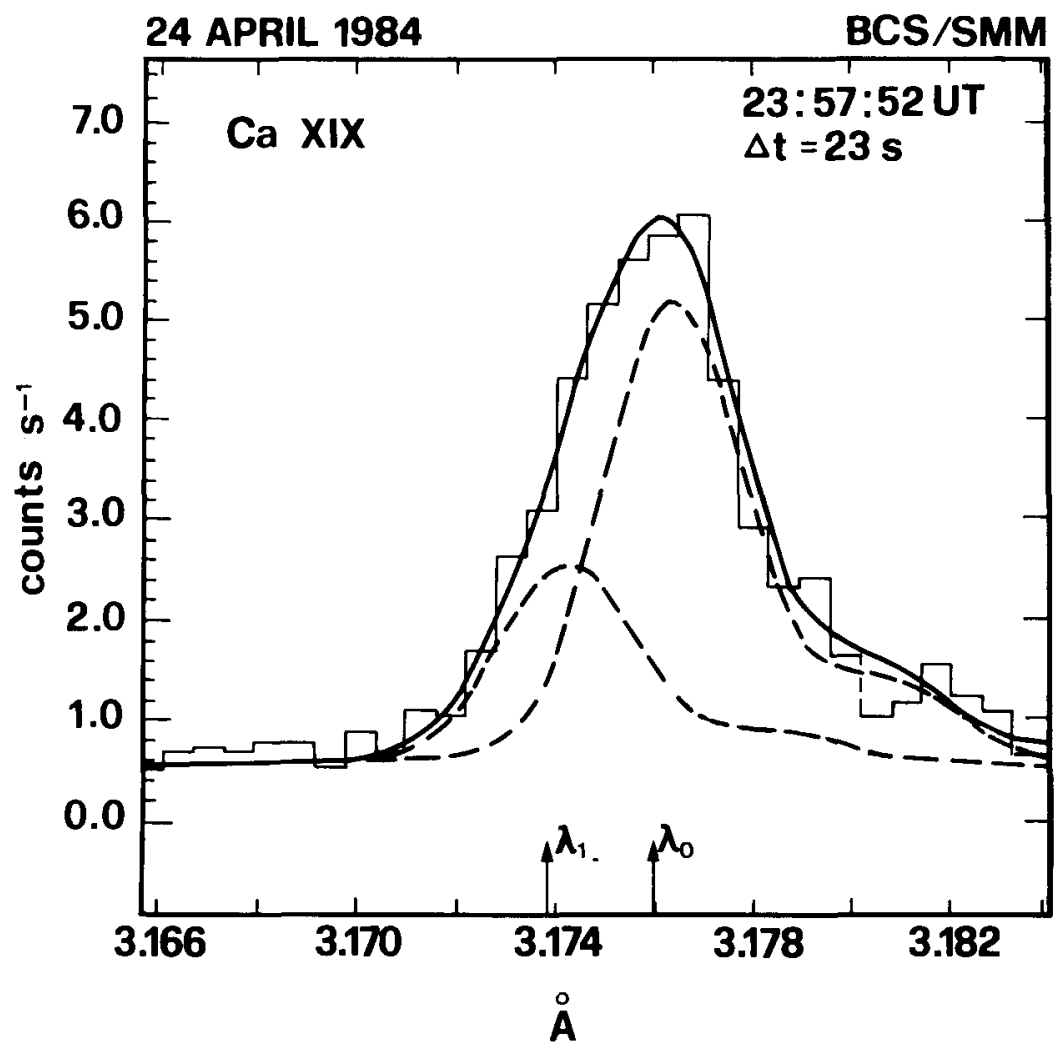

Fig. 16. CaxIX line profile (stepped line), observed during the April 24, 1984 flare, fitted with the sum (continuous line) of two spectral components (dashed lines), with relative shift consistent with a line-of-sight velocity of $210 \mathrm{~km} \mathrm{~s}^{-1}$. Bins are grouped to improve the statistics (Antonucci, Dodero, and Martin, 1989).

plasma evaporating at $v_{1}$ varies within $1.3-1.8 \times 10^{7} \mathrm{~K}$; while the lower limit derived for the higher velocity plasma varies within $1.8-3.2 \times 10^{7} \mathrm{~K}$. In conclusion, the evaporation velocity distribution during the impulsive phase of a number of large flares extends up to velocities of about $10^{3} \mathrm{~km} \mathrm{~s}^{-1}$, with a high-velocity component much hotter than the more slowly evaporating one.

The observed evaporation velocities have been compared by Fisher, Canfield, and McClymont (1984) with those obtained from hydrodynamic models in an attempt to relate the observations to the heating mechanisms operating at chromospheric level and in turn to the primary energy release. These authors have expressed the maximum values obtained in the simulated velocity distributions in units of a limiting velocity, $v_{l}$, derived with a simplified method only on the basis of a reasonable chromosphere-corona pre-flare density difference (which is assumed to be about 2.35 times the sound velocity). In Figure 18, analogous to that reported by Fisher, Canfield, and McClymont (1984), dark symbols refer to results for particle-beam models in the thick-target approximation; while, light symbols refer to thermal model results. At an energy flux of a few 


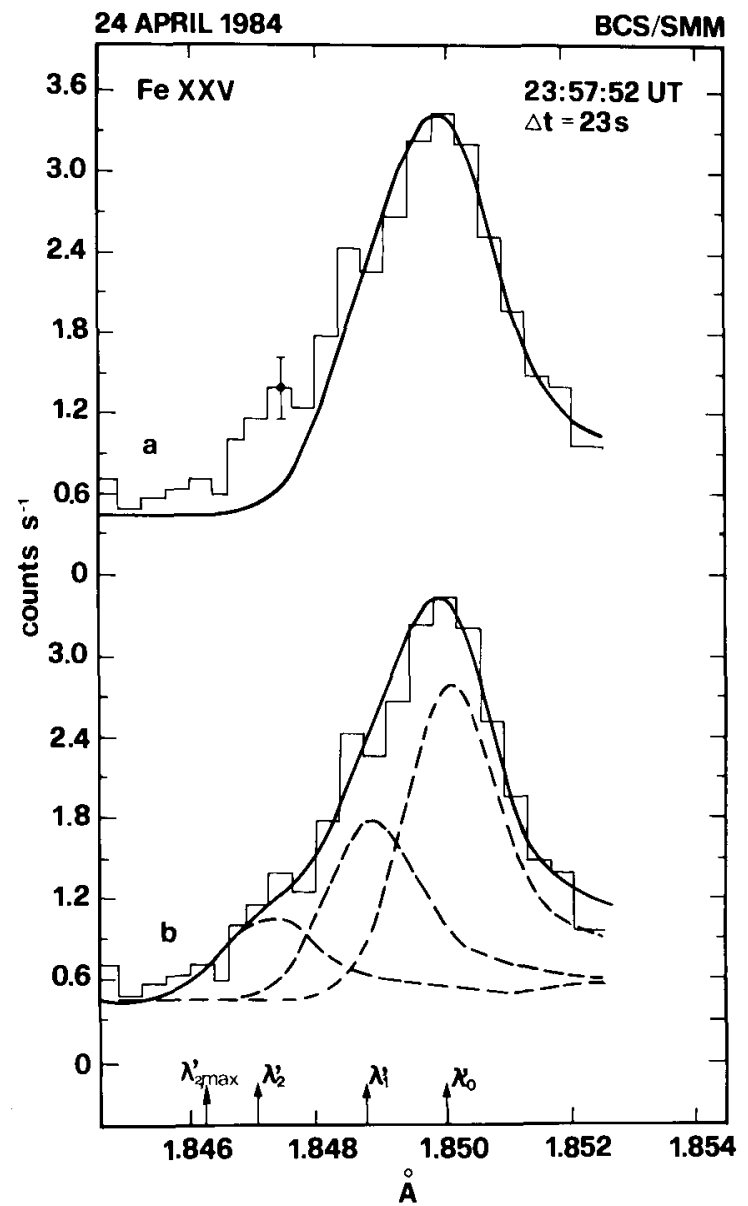

Fig. 17. Fexxv line profile (stepped line) detected, during the April 24, 1984 flare, in the high-sensitivity Bent Crystal Spectrometer channel. Fexxv line fitted with: (a) the sum (continuous line) of two spectral components with relative shift consistent with a line-of-sight velocity of $210 \mathrm{~km} \mathrm{~s}^{-1}$; (b) the superposition of an additional third spectral component with a shift consistent with a line-of-sight velocity of $480 \mathrm{~km} \mathrm{~s}^{-1}$ (Antonucci, Dodero, and Martin, 1989).

$10^{10} \mathrm{erg} \mathrm{cm}^{-2} \mathrm{~s}^{-1}$, there is the transition from 'gentle' to explosive evaporation. The velocities observed for the plasma evaporating more slowly, derived from Ca XIX spectra analysed by Antonucci, Gabriel, and Dennis (1984), cluster around the value $v_{1} / v_{l}=0.2$, or, more correctly, if we consider $v_{1, \max } / v_{l}$, maximum velocity of the slower component, around the value 0.3. On the basis of the Caxix data $\left(v_{1} / v_{l}\right)$, Fisher, Canfield, and McClymont (1984) have reached the conclusion that the observed values are consistent with 'gentle' chromospheric evaporation. The Fexxv data, however, have revealed the existence of much higher evaporation velocities, at higher plasma temperatures, which expressed as $v_{\max } / v_{l}$ fall in the interval $0.3-0.5$ (dashed region in Figure 18). This leads us to reconsider the comparison of observed and simulated results and conclude that during large flares the observed velocities are more consistent with explosive evapo- 


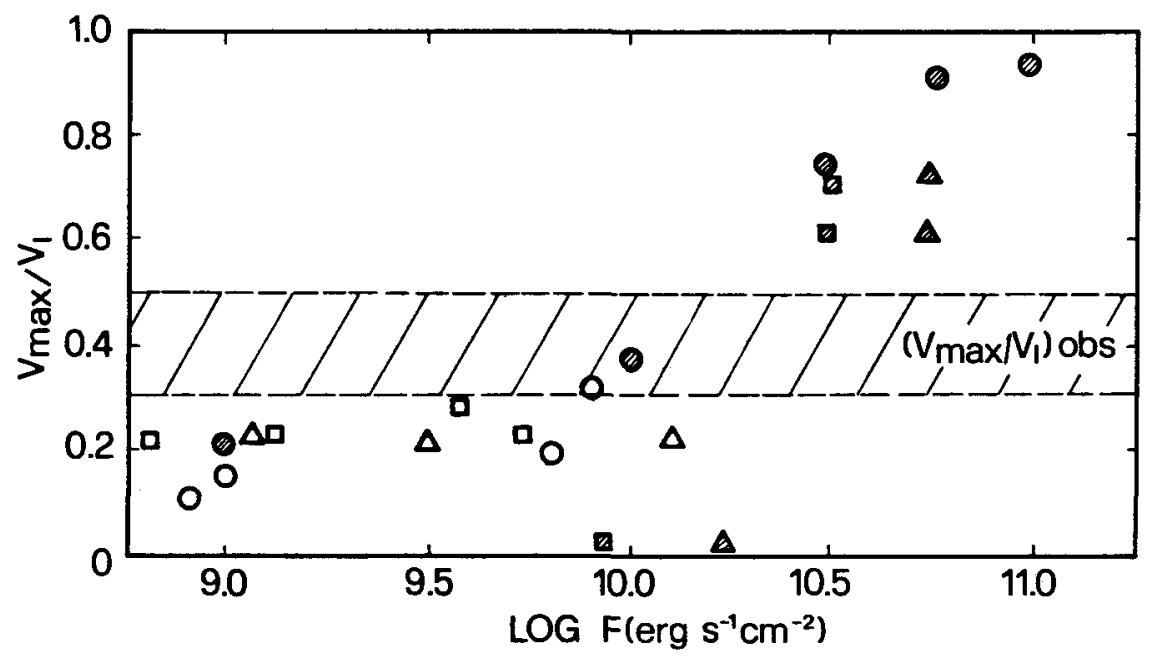

Fig. 18. Comparison of observed and simulated maximum values $v_{\max }$ in the velocity distribution of the evaporating plasma. The velocity values are expressed in units of the limiting velocity $v_{1}$ assumed equal to 235 times the sound velocity in the evaporating plasma. The dark symbols refer to thick-target particle-beam models, while light symbols refer to thermal models. The dashed region represents the interval where the observed velocities obtained with the FeXXV spectra are found. References for flare models used in this figure are reported by Fisher, Canfield, and McClymont (1984).

ration. That is, they are consistent with velocities expected for energy fluxes above $10^{10} \mathrm{erg} \mathrm{cm}^{-2} \mathrm{~s}^{-1}$. Energy fluxes into the chromosphere of this order are obtained for large flares from the observed hard X-ray emission, in the case that the source geometry is resolved (although there remain large uncertainties both in the definition of the parameters of the hard X-ray source and of the chromospheric areas interested by heating).

From the same comparison of numerical results and CaXIX observations, Fisher, Canfield, and McClymont (1984) conclude that, since none of the observed velocities exceeds the velocity limit $v_{l}$, it is not necessary to invoke additional non-hydrodynamic forces to explain the impulsive phase upflows observed in soft X-rays. This conclusion remains valid even considering the higher velocity values resulting from the FeXXV spectra (Antonucci, Dodero, and Martin, 1989). We prefer, however, to compare observations with simulated velocities independently of the velocity limit $v_{l}$, since the simulations performed for high-energy input fluxes (10 s triangular pulses with peak energy flux of $10^{11}$ ergs $\mathrm{cm}^{-2} \mathrm{~s}^{-1}$ ) by Somov, Syrovatskii, and Spektor (1981) and Somov, Sermulina, and Spektor (1982), for both thermal and non-thermal cases, lead to much higher values. Explosive evaporation at velocity exceeding $v_{l}$ is, in fact, reached in both cases for a loop length above $2 \times 10^{9} \mathrm{~cm}$. Such authors follow the evolution of the evaporating plasma for longer periods than others and their models indicate that higher evaporation velocities can be reached for more extended loops. Therefore, the observation of high velocities, consistent with those expected for explosive evaporation 
(above $10^{10} \mathrm{ergs}^{-2} \mathrm{~s}^{-1}$ in Figure 18), cannot be used to discriminate between evaporation driven by a thermal flux from a high-temperature source or by an electron beam, as it would be suggested by Figure 18, where results for thermal models are clustered in the range of energy fluxes leading to 'gentle' evaporation.

\section{Dependence of Simulation Results on the Initial Plasma Conditions}

The discussion on evaporation velocities has shown that numerical models of flaring loops can be extremely valuable for interpretating the observational results. Simulation results, however, depend not only on the heating function assumed for the loop but also on the geometry and initial conditions in the loop and, of course, on the assumptions which have been considered. Despite the simplifying assumptions used in the various numerical codes, such as one-dimensional, one-fluid, one-temperature models, the general behavior of the flare plasmas is reproduced.

As to the dependence on initial loop conditions, this becomes important when comparing simulated and observational results in individual flares. At the present the main difficulty for such a comparison concerns observations, which do not allow us to fully resolve the geometry and to determine the initial conditions in the pre-flare coronal loops. It is, however, crucial to understand the pre-flare state of loops, since there is evidence for large variations in the predicted flare physical parameters when varying initial conditions. For instance, in a model with energy deposition by an electron beam with fixed parameters, the evaporation varies significantly with the pre-flare density in a coronal loop of given geometry. Evaporation velocities drop from initial values above $700 \mathrm{~km} \mathrm{~s}^{-1}$ for an initial loop density of $2 \times 10^{9} \mathrm{~cm}^{-3}$, to peak values of about $400 \mathrm{~km} \mathrm{~s}^{-1}$, when this parameter increases to $3 \times 10^{10} \mathrm{~cm}^{-3}$ (Figure 19). This can be understood in terms both of a variation in the heating function, resulting from a variation with density in the energy losses of the energetic electrons along the loop, and of a large variation in the corona-chromosphere pressure difference. For different initial densities, the same energy input results in different temperature conditions within the loop, as shown in Figure 20. For the denser loop at $3 \times 10^{10} \mathrm{~cm}^{-3}$ the temperature peaks at the onset of energy deposition, while, for more tenuous loops temperature increases slowly to a maximum value, reached about $20 \mathrm{~s}$ after onset. The initial high temperature $\left(2.5 \times 10^{7} \mathrm{~K}\right)$ in a dense loop causes a brightening in CaXIX at the top of the loop, as shown in the simulations of the CaXIX emission along the loop, in Figure 21(a). It is interesting to note that an initial brightening at the top of the loop has been commonly considered as evidence for heating at the top of the loop. The same effect can, however, be the result of energy losses of non-thermal electrons with a relatively soft energy spectrum, in a dense environment. For the same energy input, a tenous loop brightens initially at the footpoints (Figure 21(b)). The above results are examples from a work by Antonucci et al. (1989). 


$$
\begin{gathered}
E_{c}=10 \mathrm{KeV} \\
\varphi=10^{\prime \prime} \text { ergs } \mathrm{cm}^{-2} \mathrm{sec}^{-1} \\
\partial=8
\end{gathered}
$$

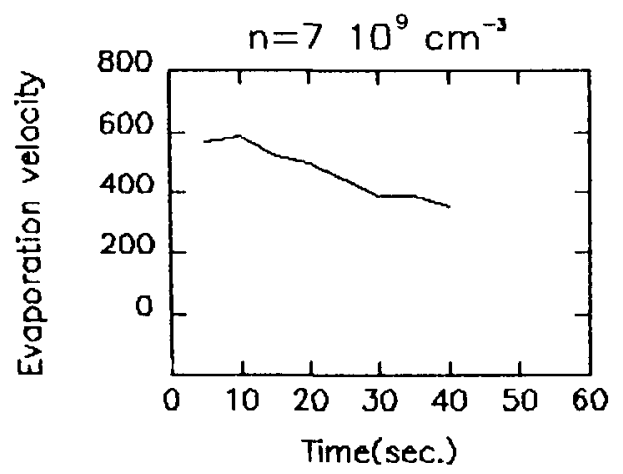

Fig. 19. Simulated averaged evaporation velocity for a non-thermal model with an electron beam represented by an energy power spectrum characterized by a cutoff $E_{\mathrm{c}}=10 \mathrm{keV}$, and a spectral index $\delta=8$. The energy flux input is constant, $\phi=10^{11} \mathrm{erg} \mathrm{cm}^{-2} \mathrm{~s}^{-1}$, over the total interval considered. The velocity is plotted for three different initial density in the coronal loop: $n_{e}=2 \times 10^{9}, 7 \times 10^{9}, 3 \times 10^{10} \mathrm{~cm}^{-3}$ (Antonucci et al., 1989).

$$
\begin{gathered}
E_{c}=10 \mathrm{KeV} \\
\varphi=10^{\prime \prime} \text { ergs } \mathrm{cm}^{-2} \mathrm{sec}^{-1} \\
\partial=8
\end{gathered}
$$

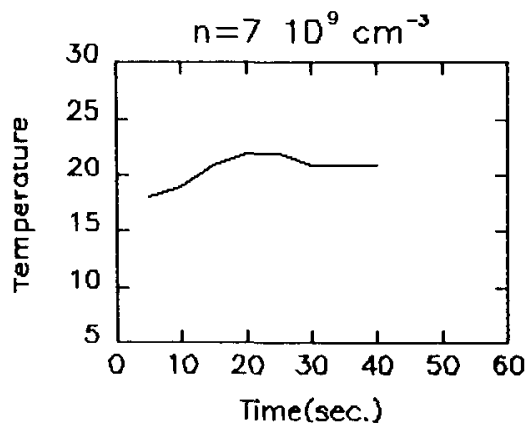

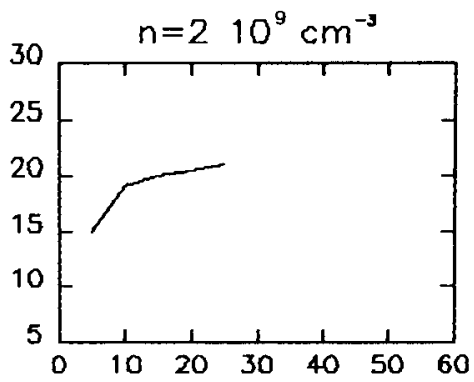

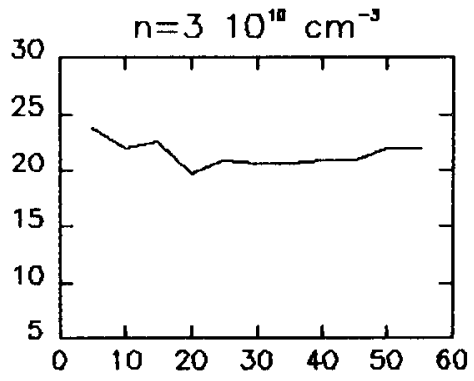

Fig. 20. Simulated average temperature (in units $10^{6} \mathrm{~K}$ ) of the flare thermal plasma for the same energy input and initial loop conditions as in Figure 19 (Antonucci et al., 1989). 


\section{Conclusions}

The major results reached during solar maximum 1980, with the advent of crystal spectroscopy at high-wavelength resolution in the soft X-ray region from 1 to $8 \AA$, can be briefly summarized in the following points.

The enhancement in non-thermal broadening observed in soft X-ray lines early in a flare can be related directly to the primary energy release process, since this effect appears to be evidence for random motions in the sites where the energy release occurs.

The convective upflows observed during the flare impulsive phase can be associated with chromospheric evaporation, and from their properties it is possible to conclude that this effect is playing a dominant role in supplying mass and energy to flaring loops and in the formation of the high-temperature plasma (above $10^{7} \mathrm{~K}$ ) characteristic of the thermal or gradual phase.

The observed 'superhot' component $\left(3-4 \times 10^{7} \mathrm{~K}\right)$ in the thermal flare plasma may turn out to be quite important for understanding the influence of the local conditions on the acceleration mechanisms.

The flare region is probably organized in a highly filamentary structure, formed by arcades of thin 'elementary' magnetic loops.

The discussion in the previous sections has focused on a few crucial areas which will be investigated in more detail and will be better understood with the advent of future soft X-ray spectrometers with higher sensitivity, which allow us to fully exploit the high temporal resolution of the spectrometers. High sensitivity and high temporal resolution

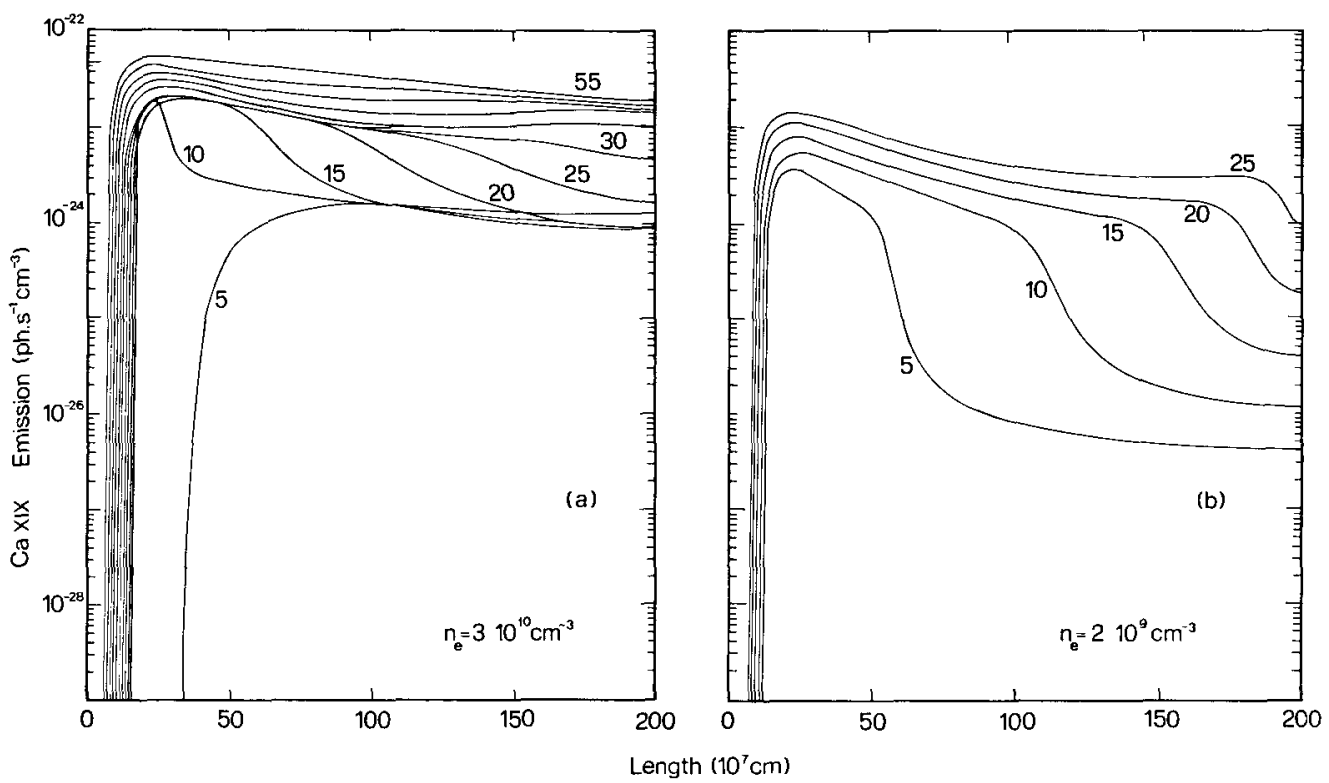

Fig. 21. Simulated Ca XIX emission along a flaring loop, with energy deposition by an electron beam with the same characteristics as in Figure 19. The initial loop density is: (a) $n_{e}=3 \times 10^{10} \mathrm{~cm}^{-3}$; (b) $n_{e}=2 \times 10^{9} \mathrm{~cm}^{-3}$ (Antonucci et al., 1989). 
are essential in the pre-onset/onset phase of flares. In fact, X-ray spectra are often well-observed when the plasma has been already heated above $10^{7} \mathrm{~K}$, and in most cases they become statistically significant, at flare onset, only when accumulated over 20-40 s. The study of flare onset is crucial in attempting: to determine the nature of linebroadenings, which in turn can give an insight into the primary energy release; to distinguish between chromospheric heating mechanisms, since the evolution of spectral profiles, as shown by simulations, depends more strongly on the energy deposition process in the initial 20-30 s of flares; and to confirm the role of the chromospheric evaporation in producing the soft X-ray thermal source during flares.

Another crucial requirement for future observations will be the capability of combining spectral and imaging observations at high spatial resolution in order to infer the initial geometry in the flaring region (possibly to confirm the filamentary nature of magnetic loops in active regions); to determine with more accuracy the initial sites of soft and hard X-ray emission during flares (this is important to understand both energy deposition and chromospheric heating mechanisms); and to attempt a more reliable measure of initial conditions in the flaring loop in order to improve the understanding of the response of the loop plasma to energy deposition.

\section{References}

Acton, L. W., Culhane, J. L., Gabriel, A. H., and 21 co-authors: 1980, Solar Phys. 65, 53.

Acton, L. W., Canfield, R. C., Gunkler, T. A., Hudson, H. S., Kiplinger, A. L., and Leibacher, J. W.: 1982, Astrophys. J. 263, 409.

Akita, K.: 1985, Ph.D. Thesis, University of Tokyo.

Antonucci, E. and Dennis, B. R.: 1983, Solar Phys. 86, 67.

Antonucci, E. and Dodero, M. A.: 1986, in D. F. Neidig (ed.), The Lower Atmosphere of Solar Flares, NSO/Sacramento Peak, p. 363.

Antonucci, E., Gabriel, A. H., and Dennis, B. R.: 1984, Astrophys. J. 287, 917.

Antonucci, E., Rosner, R., and Tsinganos, K.: 1986, Astrophys. J. 301, 975.

Antonucci, E., Rosner, R., and Tsinganos, K.: 1987, in V. E. Stepanov and V. N. Obridko (eds.), Solar Maximum Analysis, Science Press, p. 446.

Antonucci, E., Dodero, M. A., and Martin, R.: 1989, Astrophys. J. Suppl. (submitted).

Antonucci, E., Gabriel, A. H., and 7 co-authors: 1982, Solar Phys. 78, 107.

Antonucci, E., Dennis, B. R., Gabriel, A. H., and Simnett, G. M.: 1985, Solar Phys. 96, 129.

Antonucci, E., Dodero, M. A., Peres, G., Serio, S., and Rosner, R.: 1987, Astrophys. J. 322, 522.

Antonucci, E., Dodero, M. A., Peres, G., Reale, F., and Serio, S.: 1989 (in preparation).

Bhalla, C. P., Gabriel, A. H., and Presnyakov, L. P.: 1975, Monthly Notices Roy. Astron. Soc. $172,359$.

Bornmann, P. L.: 1987, Astrophys. J. 313, 449.

Bruner, M. E., Crannell, C. J., Goetz, F., Magun, A., and McKenzie, D. L.: 1988, NASA Technical Memorandum 87815.

Canfield, R. C.: 1986, Adv. Space Res. 6, 167.

Canfield, R. C., Gunkler, T. A., and Ricchiazzi, P. J.: 1985, Astrophys. J. 282, 296.

Canfield, R. C., Zarro, D. M., Metcalf, T. R., and Lemen, J. R.: 1989, Astrophys. J. (submitted).

Cheng, C.-C., Karpen, J. T., and Doschek, G. A.: 1984, Astrophys. J. 286, 787.

de Jager, C.: 1985, Solar Phys. 98, 267.

Doschek, G. A.: 1983, Solar Phys. 86, 9.

Doschek, G. A., Feldman, U., Landecker, P. B., and McKenzie, D. L.: 1981, Astrophys. J. $249,372$.

Doschek, G. A. et al: 1986, NASA Conference Publ. 2439, 4-1/4-42. 
Dubau, J., Gabriel, A. H., Loulergue, M., Steenman-Clark, L., and Volonté, S.: 1981, Monthly Notices Roy. Astron. Soc. 195, 705.

Emslie, A. G. and Alexander, D.: 1987, Solar Phys. 110, 295.

Feldman, V., Doschek, G. A., Kreplin, R. W., and Mariska, J. T.: 1980, Astrophys. J. $241,1175$.

Fisher, G. H., Canfield, R. C., and McClymont, A. N.: 1984, Astrophys. J. 281, L79.

Fisher, G. H., Canfield, R. C., and McClymont, A. N.: 1985, Astrophys. J. 289, 434.

Gabriel, A. H.: 1972, Monthly Notices Roy. Astron. Soc. 160, 99.

Gabriel, A. H. and Jordan, C.: 1969, Monthly Notices Roy. Astron. Soc. 145, 241.

Grineva, Y. I., Karev, V. I., Korneev, V. V., Krutov, V. V., Mandelstam, S. L., Vainstein, L. A., Vasilyev, B. N., and Zhitnik, I. A.: 1973, Solar Phys. 29, 441.

Gunkler, T. A., Acton, L. W., Canfield, R. C., and Kiplinger, A. L.: 1984, Astrophys. J. $285,835$.

Hiei, E. and Widing, K. G.: 1979, Solar Phys. 61, 407.

Ichimoto, K. and Kurokawa, K.: 1984, Solar Phys. 93, 105.

Karpen, J. T., Doschek, G. A., and Seely, J. F.: 1986, Astrophys. J. 306, 327.

Lin, R. P., Schwartz, R. A., Pelling, R. M., and Hurley, K. C.: 1981, Astrophys. J. 251, L109.

Linford, G. A. and Wolfson, C. J.: 1989, Astrophys. J. (in press).

Linford, G. A., Lemen, J. R., and Strong, K. T.: 1989, Adv. Space Res. (in press).

Martens, P. C. H., Van den Oord, G. H. J., and Hoyng, P.: 1985, Solar Phys. 96, 253.

McKenzie, D. L., Broussard, R. M., Landecker, P. B., Rugge, H. R., Young, R. M., Doschek, G. A., and Feldman, U.: 1980, Astrophys. J. 238, L43.

MacNeice, P.: 1986, Solar Phys. 103, 47.

MacNeice, P., McWhirter, R. W. P., Spicer, D. S., and Burgess, A.: 1984, Solar Phys. 90, 357.

Nagai, F. and Emslie, A. G.: 1984, Astrophys. J. 279, 896.

Neupert, W. M., Gates, W., Swartz, M., and Young, R.: 1967, Astrophys. J. 149, L79.

Parker, E. N.: 1983, Geophys. Astrophys. Fluid Dyn. 24, 79.

Parker, E. N.: 1987, Solar Phys. 111, 297.

Parmar, A. N., Culhane, J. L., Rapley, C. G., Antonucci, E., Gabriel, A. H., and Loulergue, M.: 1981, Monthly Notices Roy. Astron. Soc. 197, 29.

Saba, J. L. R. et al:: 1989 (in preparation).

Smith, D. F. and Harmony, D. W.: 1982, Astrophys. J. 252, 800.

Somov, B. V., Syrovatskii, S. I., and Spektor, A. R.: 1981, Solar Phys. 73, 145.

Somov, B. V., Sermulina, B. J., and Spektor, A. R.: 1982, Solar Phys. 81, 281.

Sweet, P. A.: 1969, Ann. Rev. Astron. Astrophys. 7, 149.

Takakura, T. et al.: 1983, Astrophys. J. 270, L83.

Tanaka, K.: 1986, Publ. Astron. Soc. Japan 38, 225.

Tanaka, K.: 1987, Publ. Astron. Soc. Japan 39, 1.

Tanaka, K. and Zirin, H.: 1985, Astrophys. J. 299, 1036.

Tanaka, K., Watanabe, T., Nishi, K., and Akita, K.: 1982a, Astrophys. J. 254, L59.

Tanaka, K., Akita, K., Watanabe, T., and Nishi, K.: 1982b, Hinotori Symposium on Solar Flares, Tokyo, p. 43. Tsuneta, S.: 1987, Solar Phys. 113, 35.

Tsuneta, S., Nitta, N., Ohki, K., Takakura, T., Tanaka, K., and 4 co-authors: 1984, Astrophys. J. $284,827$. Uchida, Y. and Shibata, K.: 1988, Solar Phys. 116, 291.

Watanabe, T.: 1987, Solar Phys. 113, 107.

Winglee, R. M., Pritchett, P. L., and Dulk, G. A.: 1989, Astrophys. J. (in press).

Wolfson, C. J., Doyle, J. G., Leibacher, J. W., and Phillips, K. J. H.: 1983, Astrophys. J. 269, 319.

Zarro, D. M., Strong, K. T., Canfield, R. C., Metcalf, T., and Saba, J. L. R.: 1989, Astrophys. J. (submitted). 